\title{
Second-order partition function of a non-interacting chiral fluid in $3+1$ dimensions
}

\author{
Eugenio Megías $^{a}$ and Manuel Valle ${ }^{b}$ \\ ${ }^{a}$ Grup de Física Teòrica and IFAE, Departament de Física, Universitat Autònoma de Barcelona, \\ Bellaterra 08193 Barcelona, Spain \\ ${ }^{b}$ Departamento de Física Teórica, Universidad del País Vasco UPV/EHU, \\ Apartado 644, 48080 Bilbao, Spain \\ E-mail: emegias@ifae.es, manuel.valle@ehu.es
}

ABSTRACT: We compute the partition function for non-interacting chiral fermions at second order in a derivative expansion of an arbitrary time-independent gravitational and gauge background. We find that Pauli-Villars regularization of the vacuum part is needed to get consistent results. We use our results to discuss some features of the non-dissipative constitutive relations of second order hydrodynamics.

Keywords: Thermal Field Theory, Holography and quark-gluon plasmas, Anomalies in Field and String Theories

ArXiv ePrint: 1408.0165 


\section{Contents}

1 Introduction 1

2 Theory of free Dirac fermions: U(1) current and energy-momentum tensor

3 The Green function $\quad 5$

$\begin{array}{ll}3.1 \text { Green function at leading order } & 6\end{array}$

$\begin{array}{ll}3.2 \text { Green function at higher derivative orders } & 6\end{array}$

4 Covariant current and stress tensor at first order $\quad 7$

5 Energy and charge density at second order. Renormalization 9

$\begin{array}{lll}5.1\left\langle T_{00}\right\rangle \text { and }\left\langle J_{0}\right\rangle \text { for Weyl fermions } & 10\end{array}$

5.2 Vacuum expectation values from regulators: Pauli-Villars regularization 11

$6 \quad$ Partition function at second order $\quad 12$

7 Non-dissipative constitutive relations from the partition function $\quad 16$

8 Conclusion $\quad 21$

A Free theory of Dirac fermions $\quad 22$

B Technical details on the computation of the thermal expectation values:

$\begin{array}{ll}\text { Matsubara sums } & \mathbf{2 3}\end{array}$

\section{Introduction}

One of the most fruitful techniques to study physical systems out of equilibrium is the hydrodynamical approach, in which it is assumed that the scales of variation of its observables are much longer than any microphysical scale in the system (see e.g. [1] for a review). The key ingredients to study the hydrodynamical systems are the so-called constitutive relations, which are expressions relating the conserved currents of the systems, energymomentum tensor and charged currents, with fluid variables like temperature, chemical potential, and fluid velocity. The hydrodynamical approach organizes the constitutive relations in a derivative expansion of the fluid variables, and the various terms appearing in this expansion are multiplied by transport coefficients or susceptibilities. Some of these coefficients are responsible for dissipative effects, as they induce an entropy production in the system out of equilibrium. Examples of dissipative coefficients at first order in the hydrodynamical expansion are the shear viscosity $\eta$ and bulk viscosity $\zeta[1,2]$. Other kind 
of coefficients in the constitutive relations is related to the static response of the system to an external perturbation, and they can be obtained from the equilibrium properties. The magnetic susceptibility pertains to this kind.

In the past few years a new set of transport coefficients induced by chiral anomalies has received much attention and interest. In presence of anomalies the currents are no longer conserved, and this has important effects in the constitutive relations. Some examples of anomalous coefficients at first order are the chiral magnetic conductivity, which is responsible for the generation of an electric current parallel to a strong magnetic field in the system [3], and the chiral vortical conductivity, in which the electric current is induced by a vortex [4]. These conductivities are almost completely fixed by imposing the requirement of zero entropy production in the equation for the divergence of the entropy current.

Recently, it has been shown in $[5,6]$ that it is not necessary to resort to entropy arguments to obtain the non-dissipative part of the anomalous constitutive relations. The existence of a local partition function that reproduces the consistent currents in stationary conditions is all that is needed. It turns out that the determination of the most general partition function in a stationary background becomes an important issue not only with regard to the thermodynamics, but also with hydrodynamics (see [7] for considerations concerning the construction of an entropy current from the partition function). Other methods to compute the transport coefficients from a microscopic theory include kinetic theory [8-10], Kubo formulae [11] and fluid/gravity correspondence [12]. In particular, the second method allowed the identification of a purely temperature dependent contribution in the chiral vortical conductivity not determined by the second law of thermodynamics, and it was shown to arise when the system features a mixed gauge-gravitational anomaly $[13,14]$. This was later confirmed by other methods [15-19].

In order to gain more insight into the effects of anomalies, it may be of interest to go to higher orders in the hydrodynamical derivative expansion. In this paper we do this by considering the manageable problem of an ideal fluid of chiral fermions. The main goal is the computation of the partition function at second order in the derivative expansion for this system. A classification of terms contributing to this order in the constitutive relations was done in [20]. Following [5], we have considered an arbitrary time-independent background given by the line element and $\mathrm{U}(1)$ gauge connection

$$
\begin{aligned}
d s^{2} & =G_{\mu \nu} d x^{\mu} d x^{\nu}=-e^{2 \sigma(\boldsymbol{x})}\left(d t+a_{i}(\boldsymbol{x}) d x^{i}\right)^{2}+g_{i j}(\boldsymbol{x}) d x^{i} d x^{j}, \\
\mathcal{A}_{\mu} & =\left(A_{0}(\boldsymbol{x}), \boldsymbol{\mathcal { A }}(\boldsymbol{x})\right) .
\end{aligned}
$$

It is convenient to introduce the combination $A_{i} \equiv \mathcal{A}_{i}-A_{0} a_{i}$, which is invariant under the Kaluza-Klein gauge transformation given by the time reparametrization $t \rightarrow t+\phi(\boldsymbol{x})$, $\boldsymbol{x} \rightarrow \boldsymbol{x}$. The most general parity even partition function to second order in the derivative expansion is built from seven scalar and two pseudo-scalar quantities as follows [21, 22]

$$
\begin{aligned}
\mathcal{W}_{2}=\int d^{3} x \sqrt{g}[ & M_{1}\left(\sigma, A_{0}\right) T_{0}^{2} e^{-2 \sigma} \nabla^{i} \sigma \nabla_{i} \sigma+\frac{M_{2}\left(\sigma, A_{0}\right)}{T_{0}^{2}} \nabla^{i} A_{0} \nabla_{i} A_{0} \\
& -M_{3}\left(\sigma, A_{0}\right) e^{-\sigma} \nabla^{i} \sigma \nabla_{i} A_{0}+T_{0}^{2} M_{4}\left(\sigma, A_{0}\right) f_{i j} f^{i j}+M_{5}\left(\sigma, A_{0}\right) F_{i j} F^{i j}
\end{aligned}
$$




$$
\begin{gathered}
\left.+T_{0} M_{6}\left(\sigma, A_{0}\right) f_{i j} F^{i j}+M_{7}\left(\sigma, A_{0}\right) R\right] \\
+\int d^{3} x \sqrt{g}\left[N_{1}\left(\sigma, A_{0}\right) \epsilon^{i j k} \partial_{i} A_{0} f_{j k}+N_{2}\left(\sigma, A_{0}\right) \epsilon^{i j k} \partial_{i} A_{0} F_{j k}\right],
\end{gathered}
$$

where $T_{0}^{-1}$ is the period of the imaginary time, $R$ is the three-dimensional Ricci scalar from $g_{i j}$, and we have defined the strength tensors $F_{i j}=\partial_{i} A_{j}-\partial_{j} A_{i}$ and $f_{i j}=\partial_{i} a_{j}-\partial_{j} a_{i}$. The functions $M_{i}\left(\sigma, A_{0}\right), N_{j}\left(\sigma, A_{0}\right), i=1, \ldots, 7, j=1,2$, depend on the specific system. Here, we have computed them for an ideal gas of Weyl and Dirac fermions. We find that the parity odd part of the partition function parametrized by the $N_{j}$ vanishes in the absence of time reversal symmetry breaking. After using Pauli-Villars regularization, we identify the terms in the partition function related to the trace anomaly. We also obtain partially the form of non-dissipative constitutive relations in the Landau frame by keeping only the terms of second derivatives of the fluid and background fields.

The manuscript is organized as follows. In section 2 we obtain the expressions of the charged U(1) current and energy-momentum tensor for a theory of free Dirac fermions in $3+1$ dimensions in terms of the thermal Green function. Then, we compute in section 3 the Green function up to second order in derivatives of the background fields. With these results we obtain in section 4 the equilibrium expectation values of the current and energymomentum tensor as well as the partition function at first order, and in section 5 the energy and charge density at second order. We present in section 6 our result for the equilibrium partition function at second order, and this is used in section 7 to compute the non-dissipative part of the second order constitutive relations in the parity even sector. Finally we conclude with a discussion of our results in section 8.

\section{Theory of free Dirac fermions: U(1) current and energy-momentum tensor}

Our main goal in this manuscript is to determine the partition function of free chiral fermions in $3+1$ dimensions up to second order in the derivatives of the metric and gauge background fields. However, as we explain in section 5.2, we will derive the result by using Pauli-Villars regularization, and this demands the consideration of the massive theory for the vacuum contribution. So, for the sake of completeness we develop in sections 2 and 3 the formalism for a massive Dirac field. We keep in appendix A some technical details of this formalism.

Since the partition function will be computed from the equilibrium values of the $\mathrm{U}(1)$ current and the stress tensor, we begin with the expressions

$$
\begin{aligned}
J^{\mu} & =-\bar{\Psi} \underline{\gamma}^{\mu} \Psi \\
T_{\mu \nu} & =\frac{i}{4} \bar{\Psi}\left[\underline{\gamma}_{\mu} \vec{\nabla}_{\nu}-\overleftarrow{\nabla}_{\nu} \underline{\gamma}_{\mu}+(\mu \leftrightarrow \nu)\right] \Psi,
\end{aligned}
$$

where it has been assumed that the spinor field satisfies the Dirac equation. The left and right currents are defined by $J_{L, R}^{\mu}=-\bar{\Psi} \underline{\gamma}^{\mu} \mathcal{P}_{L, R} \Psi$, where $\mathcal{P}_{L, R}=\frac{1}{2}\left(1 \pm \gamma_{5}\right)$ are the chiral 
projectors. Using the explicit form of the background we have

$$
\begin{aligned}
J_{0}= & -e^{-\sigma} \psi^{\dagger} \psi, \\
J^{i}= & -\psi^{\dagger} \sigma_{i} \psi, \\
T_{00}= & \frac{i}{2} e^{\sigma}\left(\psi^{\dagger} \partial_{t} \psi-\partial_{t} \psi^{\dagger} \psi\right)+e^{\sigma} A_{0} \psi^{\dagger} \psi-\frac{1}{4} e^{3 \sigma} \epsilon^{i j k} \partial_{j} a_{k} \psi^{\dagger} \sigma_{i} \psi, \\
T_{0}^{i}= & \frac{i}{4} e^{\sigma}\left(\psi^{\dagger} \partial_{i} \psi-\partial_{i} \psi^{\dagger} \psi\right)-\frac{i}{4} e^{\sigma} a_{i}\left(\psi^{\dagger} \partial_{t} \psi-\partial_{t} \psi^{\dagger} \psi\right)+\frac{i}{4}\left(\psi^{\dagger} \sigma_{i} \partial_{t} \psi-\partial_{t} \psi^{\dagger} \sigma_{i} \psi\right) \\
& +\frac{1}{2} e^{\sigma}\left(\mathcal{A}_{i}-a_{i} A_{0}\right) \psi^{\dagger} \psi-\frac{1}{8} e^{2 \sigma} \epsilon^{i j k} \partial_{j} a_{k} \psi^{\dagger} \psi+\frac{1}{4} e^{\sigma} \epsilon^{i j k} \partial_{j} \sigma \psi^{\dagger} \sigma_{k} \psi+\frac{1}{2} A_{0} \psi^{\dagger} \sigma_{i} \psi,
\end{aligned}
$$

where eqs. (2.2) and (2.3) correspond to the left-handed current, and $\psi$ is the twocomponent Weyl fermion $\psi_{L}$, cf. eq. (A.3). Similarly, eqs. (2.4) and (2.5) are the contributions to the stress tensor from the left-handed part. The inclusion of right-handed fermions in these formulas is straightforward. We omit in the following the subindex $L$ to simplify the notation.

The expectation values of these quantities at equilibrium may be computed from the thermal Green's function defined as

$$
\left\langle T \psi(-i \tau, \boldsymbol{x}) \psi^{\dagger}\left(0, \boldsymbol{x}^{\prime}\right)\right\rangle_{\beta}=T_{0} \sum_{n} e^{-i \omega_{n} \tau} \mathcal{G}\left(\boldsymbol{x}, \boldsymbol{x}^{\prime}, \omega_{n}\right)
$$

where $\omega_{n}=\frac{2 \pi}{\beta}\left(n+\frac{1}{2}\right)$ are the fermionic Matsubara frequencies and $\beta=1 / T_{0}$. The precise form of these is

$$
\begin{aligned}
& \left\langle J_{0}\right\rangle=T_{0} \sum_{n}\left[-e^{\sigma} \operatorname{tr} \mathcal{G}\left(\boldsymbol{x}, \boldsymbol{x}, \omega_{n}\right)\right], \\
& \left\langle J^{i}\right\rangle=-T_{0} \sum_{n} \operatorname{tr}\left[\sigma_{i} \mathcal{G}\left(\boldsymbol{x}, \boldsymbol{x}, \omega_{n}\right)\right],
\end{aligned}
$$

and

$$
\begin{aligned}
\left\langle T_{00}\right\rangle= & T_{0} \sum_{n}\left[e^{\sigma}\left(i \omega_{n}+A_{0}\right) \operatorname{tr} \mathcal{G}\left(\boldsymbol{x}, \boldsymbol{x}, \omega_{n}\right)-\frac{1}{4} e^{3 \sigma} \epsilon^{i j k} \partial_{j} a_{k} \operatorname{tr}\left[\sigma_{i} \mathcal{G}\left(\boldsymbol{x}, \boldsymbol{x}, \omega_{n}\right)\right]\right], \\
\left\langle T_{0}^{i}\right\rangle= & \left.T_{0} \sum_{n}\left[\frac{i}{4} e^{\sigma} \operatorname{tr}\left(\frac{\partial}{\partial x^{i}} \mathcal{G}\left(\boldsymbol{x}, \boldsymbol{x}^{\prime}, \omega_{n}\right)-\frac{\partial}{\partial x^{\prime i}} \mathcal{G}\left(\boldsymbol{x}, \boldsymbol{x}^{\prime}, \omega_{n}\right)\right)\right]\right|_{\boldsymbol{x}^{\prime}=\boldsymbol{x}} \\
& +T_{0} \sum_{n}\left[\frac{1}{2} e^{\sigma} A_{i} \operatorname{tr} \mathcal{G}\left(\boldsymbol{x}, \boldsymbol{x}, \omega_{n}\right)+\frac{1}{4} e^{\sigma} \epsilon^{i j k} \partial_{j} \sigma \operatorname{tr}\left[\sigma_{k} \mathcal{G}\left(\boldsymbol{x}, \boldsymbol{x}, \omega_{n}\right)\right]\right] \\
& +T_{0} \sum_{n}\left[\frac{1}{2}\left(i \omega_{n}+A_{0}\right) \operatorname{tr}\left[\sigma_{i} \mathcal{G}\left(\boldsymbol{x}, \boldsymbol{x}, \omega_{n}\right)\right]-\frac{1}{2} e^{\sigma} a_{i}\left(i \omega_{n}+A_{0}\right) \operatorname{tr} \mathcal{G}\left(\boldsymbol{x}, \boldsymbol{x}, \omega_{n}\right)\right] \\
& +T_{0} \sum_{n}\left[-\frac{1}{8} e^{2 \sigma} \epsilon^{i j k} \partial_{j} a_{k} \operatorname{tr} \mathcal{G}\left(\boldsymbol{x}, \boldsymbol{x}, \omega_{n}\right)\right] .
\end{aligned}
$$


Therefore, the partition function $\mathcal{W}$ may be determined by integration of the variational formulae [5]

$$
\begin{aligned}
\left\langle J^{i}\right\rangle & =\frac{T_{0}}{\sqrt{-G}} \frac{\delta \mathcal{W}}{\delta A_{i}}, & \left\langle J_{0}\right\rangle & =-\frac{T_{0} e^{2 \sigma}}{\sqrt{-G}} \frac{\delta \mathcal{W}}{\delta A_{0}} \\
\left\langle T_{0}^{i}\right\rangle & =\frac{T_{0}}{\sqrt{-G}}\left(\frac{\delta \mathcal{W}}{\delta a_{i}}-A_{0} \frac{\delta \mathcal{W}}{\delta A_{i}}\right), & \left\langle T_{00}\right\rangle & =-\frac{T_{0} e^{2 \sigma}}{\sqrt{-G}} \frac{\delta \mathcal{W}}{\delta \sigma} .
\end{aligned}
$$

Note that a variation of $\mathcal{W}_{2}$ in eq. (1.2) with respect to $\sigma$ or $A_{0}$ always produces terms which are a product of two first order derivatives. Thus, in order to obtain the form of the coefficients $M_{i}\left(\sigma, A_{0}\right)$ it is sufficient to determine such bilinear contributions in $\left\langle T_{00}\right\rangle$ and $\left\langle J_{0}\right\rangle$. Clearly, the first six coefficients may be computed by setting the metric flat, $g_{i j}=\delta_{i j}$, but the determination of $M_{7}$ demands the computation of the energy density to first order in the three-dimensional curvature $R$.

In the next section we will compute the Green function as an expansion in derivatives of the background fields. After that, we will use the expressions above to compute the thermal expectation value of the charged current and energy-momentum tensor at equilibrium at first order, and the charge and energy density at second order.

\section{The Green function}

There are several ways to compute the two-point Green function. We will follow the procedure of ref. [23]. We can rewrite the action as

$$
S=-\int d^{4} x \sqrt{-G} \bar{\Psi} \underline{\gamma}^{0}\left[i \partial_{t}-\mathcal{H}\right] \Psi
$$

with the Hamiltonian

$$
\mathcal{H}=-i\left(\frac{1}{4} \omega_{0}^{a b} \gamma_{a b}-i A_{0}\right)-\frac{i}{g^{00}} \underline{\gamma}^{0}\left(\underline{\gamma}^{k} \nabla_{k}-m\right)
$$

After rotating to imaginary time $t \rightarrow-i \tau$, the Green function satisfies the differential equation

$$
-\sqrt{-G} \gamma^{0} \underline{\gamma}^{0}\left(i \omega_{n}-\mathcal{H}\right) \mathcal{G}\left(\boldsymbol{x}, \boldsymbol{x}^{\prime}, \omega_{n}\right)=\delta\left(\boldsymbol{x}-\boldsymbol{x}^{\prime}\right) .
$$

The Hamiltonian does not depend on terms beyond first order in derivatives of the background fields. After some algebra one gets the exact equation for the Green function

$$
\left[\left(\mathbb{1}_{4 \times 4}+e^{\sigma(\boldsymbol{x})} \gamma^{0} \boldsymbol{\gamma} \cdot \boldsymbol{a}(\boldsymbol{x})\right) i \omega_{n}-H(\boldsymbol{x})\right] \mathcal{G}\left(\boldsymbol{x}, \boldsymbol{x}^{\prime}, \omega_{n}\right)=\delta^{(3)}\left(\boldsymbol{x}-\boldsymbol{x}^{\prime}\right),
$$

where $H(\boldsymbol{x})=H_{0}(\boldsymbol{x})+H_{1}(\boldsymbol{x})$ with

$$
\begin{aligned}
& H_{0}=-A_{0} \mathbb{1}_{4 \times 4}-i e^{\sigma} m \gamma^{0}+e^{\sigma} \gamma^{0} \boldsymbol{\gamma} \cdot\left(i \boldsymbol{\partial}+\mathcal{A}-A_{0} \boldsymbol{a}\right), \\
& H_{1}=\frac{i}{2} e^{\sigma} \gamma^{0} \boldsymbol{\gamma} \cdot \boldsymbol{\partial} \sigma+\frac{i}{4} e^{2 \sigma} \gamma^{j k} \partial_{j} a_{k},
\end{aligned}
$$

and we have defined $\gamma^{j k}=\frac{1}{2}\left[\gamma^{j}, \gamma^{k}\right]$. Note that eq. (3.4) is Kaluza-Klein gauge invariant, as it depends on the combination $\mathcal{A}_{i}-A_{0} a_{i}$. In addition, the term proportional to $i e^{\sigma} \gamma^{0} \boldsymbol{\gamma} \cdot \boldsymbol{\partial}$ 
of $H_{0}$ in combination with the term $i e^{\sigma} \gamma^{0} \boldsymbol{\gamma} \cdot \boldsymbol{\partial}$ of $H_{1}$ guarantees the hermiticity of the operator $H$.

This equation can be solved order by order in a derivative expansion of the background fields. The solution for the Green function will be of the form $\mathcal{G}=\mathcal{G}_{0}+\mathcal{G}_{1}+\mathcal{G}_{2}+\ldots$, where the subscript indicates the order in derivatives.

\subsection{Green function at leading order}

The Green function at leading order is obtained by neglecting $H_{1}$ in eq. (3.4) and evaluating the background fields at a reference point $\boldsymbol{z}$. After Fourier transforming this equation, one can solve it easily as explained in ref. [23]. The result is

$$
\begin{aligned}
\mathcal{G}_{0}\left(\boldsymbol{x}, \boldsymbol{x}^{\prime}, \omega_{n}\right)=-\frac{i}{16 \pi^{3 / 2}} e^{i\left(\boldsymbol{\mathcal { A }}-\left(A_{0}+i \omega_{n}\right) \boldsymbol{a}\right) \cdot\left(\boldsymbol{x}-\boldsymbol{x}^{\prime}\right)-2 \sigma} \int_{0}^{\infty} \frac{d s}{s^{5 / 2}} e^{-\frac{\left|\boldsymbol{x}-\boldsymbol{x}^{\prime}\right|}{4 s}+b^{2} s} \\
\times\left(-2 s\left[\left(A_{0}+i \omega_{n}\right) \mathbb{1}_{4 \times 4}-i m e^{\sigma} \gamma^{0}\right]+i e^{\sigma} \gamma^{0} \gamma^{i}\left(x^{i}-x^{i}\right)\right),
\end{aligned}
$$

where

$$
b^{2}=-m^{2}+e^{-2 \sigma}\left(A_{0}+i \omega_{n}\right)^{2} .
$$

For computational convenience in what follows, we have made use of the proper time representation. This allows to transform the integrals in the space coordinates $\boldsymbol{x}$ and $\boldsymbol{x}^{\prime}$, into Gaussian integrals that are much more analytically treatable.

\subsection{Green function at higher derivative orders}

We will study next the solution of eq. (3.4) at first and second order in the derivative expansion. We consider an expansion of the background fields around the reference point $\boldsymbol{z}$, i.e.

$$
\Xi(\boldsymbol{x})=\Xi(\boldsymbol{z})+\left(x^{i}-z^{i}\right) \partial_{i} \Xi(\boldsymbol{z})+\frac{1}{2}\left(x^{i}-z^{i}\right)\left(x^{j}-z^{j}\right) \partial_{i} \partial_{j} \Xi(\boldsymbol{z})+\ldots,
$$

where $\Xi \equiv \sigma, A_{0}, \mathcal{A}_{k}, a_{k}$. Then $H(x)$ has the following expansion

$$
H(\boldsymbol{x})=H_{0}(\boldsymbol{z})+\delta_{1} H(\boldsymbol{x})+\delta_{2} H(\boldsymbol{x})+\ldots,
$$

where the first and second derivative contributions are, respectively,

$$
\begin{aligned}
& \delta_{1} H(\boldsymbol{x})=\left.\left(x^{i}-z^{i}\right) \partial_{i} H_{0}\right|_{z}+H_{1}(\boldsymbol{z}), \\
& \delta_{2} H(\boldsymbol{x})=\left.\frac{1}{2}\left(x^{i}-z^{i}\right)\left(x^{j}-z^{j}\right) \partial_{i} \partial_{j} H_{0}\right|_{z}+\left.\left(x^{i}-z^{i}\right) \partial_{i} H_{1}\right|_{z} .
\end{aligned}
$$

The expansion of the factor $e^{\sigma(\boldsymbol{x})} \gamma^{0} \boldsymbol{\gamma} \cdot \boldsymbol{a}(\boldsymbol{x})$ of eq. (3.4) up to second order is $e^{\sigma(\boldsymbol{x})} \gamma^{0} \boldsymbol{\gamma} \cdot \boldsymbol{a}(\boldsymbol{x})=$ $e^{\sigma(\boldsymbol{z})} \gamma^{0} \boldsymbol{\gamma} \cdot \boldsymbol{a}(\boldsymbol{z})+\delta_{1} f(\boldsymbol{x})+\delta_{2} f(\boldsymbol{x})$, with

$$
\begin{aligned}
& \delta_{1} f(\boldsymbol{x})=\left(x^{i}-z^{i}\right) e^{\sigma(\boldsymbol{z})} \gamma^{0} \boldsymbol{\gamma} \cdot\left(\partial_{i} \boldsymbol{a}(\boldsymbol{z})+\boldsymbol{a}(\boldsymbol{z}) \partial_{i} \sigma(\boldsymbol{z})\right), \\
& \begin{aligned}
\delta_{2} f(\boldsymbol{x})=\frac{1}{2}\left(x^{i}-z^{i}\right)\left(x^{j}-z^{j}\right) e^{\sigma(\boldsymbol{z})} \gamma^{0} \boldsymbol{\gamma} \cdot( & \left(2 \partial_{i} \boldsymbol{a}(\boldsymbol{z}) \partial_{j} \sigma(\boldsymbol{z})+\partial_{i} \partial_{j} \boldsymbol{a}(\boldsymbol{z})\right. \\
& \left.+\boldsymbol{a}(\boldsymbol{z}) \partial_{i} \sigma(\boldsymbol{z}) \partial_{j} \sigma(\boldsymbol{z})+\boldsymbol{a}(\boldsymbol{z}) \partial_{i} \partial_{j} \sigma(\boldsymbol{z})\right) .
\end{aligned}
\end{aligned}
$$


Note that the background fields in these expansions are always evaluated at the reference point $\boldsymbol{z}$. Substituting the expansions eqs. (3.11)-(3.14) into eq. (3.4) yields the following differential equations for $\mathcal{G}_{1}$ and $\mathcal{G}_{2}$ respectively,

$$
\begin{aligned}
\left(i \omega_{n}-H_{0}(z)\right) \mathcal{G}_{1}\left(\boldsymbol{x}, \boldsymbol{x}^{\prime}, \omega_{n}\right) & =\left(\delta_{1} H(\boldsymbol{x})-\delta_{1} f(\boldsymbol{x}) i \omega_{n}\right) \mathcal{G}_{0}\left(\boldsymbol{x}, \boldsymbol{x}^{\prime}, \omega_{n}\right), \\
\left(i \omega_{n}-H_{0}(z)\right) \mathcal{G}_{2}\left(\boldsymbol{x}, \boldsymbol{x}^{\prime}, \omega_{n}\right) & =\left(\delta_{2} H(\boldsymbol{x})-\delta_{2} f(\boldsymbol{x}) i \omega_{n}\right) \mathcal{G}_{0}\left(\boldsymbol{x}, \boldsymbol{x}^{\prime}, \omega_{n}\right) \\
& +\left(\delta_{1} H(\boldsymbol{x})-\delta_{1} f(\boldsymbol{x}) i \omega_{n}\right) \mathcal{G}_{1}\left(\boldsymbol{x}, \boldsymbol{x}^{\prime}, \omega_{n}\right) .
\end{aligned}
$$

The solution of the Green function at second order, $\mathcal{G}_{2}$, is more involved than at first order, and demands the computation of direct and exchange terms. These equations can be solved in a Schwinger-Dyson expansion to get

$$
\begin{aligned}
\mathcal{G}_{1}\left(\boldsymbol{x}, \boldsymbol{x}^{\prime}, \omega_{n}\right)=\int d^{3} x^{\prime \prime} \mathcal{G}_{0}\left(\boldsymbol{x}, \boldsymbol{x}^{\prime \prime}, \omega_{n}\right)\left(\delta_{1} H\left(\boldsymbol{x}^{\prime \prime}\right)-\delta_{1} f\left(\boldsymbol{x}^{\prime \prime}\right) i \omega_{n}\right) \mathcal{G}_{0}\left(\boldsymbol{x}^{\prime \prime}, \boldsymbol{x}^{\prime}, \omega_{n}\right) \\
\mathcal{G}_{2}\left(\boldsymbol{x}, \boldsymbol{x}^{\prime}, \omega_{n}\right)=\int d^{3} x^{\prime \prime} \mathcal{G}_{0}\left(\boldsymbol{x}, \boldsymbol{x}^{\prime \prime}, \omega_{n}\right)\left(\delta_{2} H\left(\boldsymbol{x}^{\prime \prime}\right)-\delta_{2} f\left(\boldsymbol{x}^{\prime \prime}\right) i \omega_{n}\right) \mathcal{G}_{0}\left(\boldsymbol{x}^{\prime \prime}, \boldsymbol{x}^{\prime}, \omega_{n}\right) \\
\quad+\int d^{3} x^{\prime \prime} \mathcal{G}_{0}\left(\boldsymbol{x}, \boldsymbol{x}^{\prime \prime}, \omega_{n}\right)\left(\delta_{1} H\left(\boldsymbol{x}^{\prime \prime}\right)-\delta_{1} f\left(\boldsymbol{x}^{\prime \prime}\right) i \omega_{n}\right) \mathcal{G}_{1}\left(\boldsymbol{x}^{\prime \prime}, \boldsymbol{x}^{\prime}, \omega_{n}\right) .
\end{aligned}
$$

The evaluation of these integrals is rather lengthy, specially those for the second order Green function. Each of these integrals involves the product of two Green's functions, and requires the integration over two proper times $\int_{0}^{\infty} d s_{1} \int_{0}^{\infty} d s_{2}$. The best way to proceed is to work with new variables $\rho \equiv s_{1}+s_{2}, s_{1} \equiv \rho \xi$, so that the double integral in proper times becomes

$$
\int_{0}^{\infty} d s_{1} \int_{0}^{\infty} d s_{2} f\left(s_{1}, s_{2}\right)=\int_{0}^{\infty} d \rho \rho \int_{0}^{1} d \xi f(\rho \xi, \rho(1-\xi)) .
$$

The integrals in $\xi$ are finite and can be done straightforwardly in general, so that one ends up with expressions which have to be integrated in the parameter $\rho$. The possible appearance of divergences in the integral over $\rho$ and its regularization will be explained in detail in section 5 . The complete expressions for $\mathcal{G}_{1}$ and $\mathcal{G}_{2}$ are very lengthy and will not be presented here. Instead, we will use them in the next two sections to compute the thermal expectation values of the current and energy-momentum tensor at first and second order in derivatives.

\section{Covariant current and stress tensor at first order}

After obtaining the thermal Green function at first order, we can compute the U(1) current and energy-momentum tensor at this order by using eqs. (2.7)-(2.10). In the following we will restrict ourselves to a theory with one left Weyl fermion. We are focusing on the parityodd contributions. In order to compute the result at first order in derivatives, we need to evaluate each term of these equations to the appropriate order in the Green function. In particular, the formulas for $\left\langle J^{i}\right\rangle$ and $\left\langle T_{0}{ }^{i}\right\rangle$ become

$$
\begin{aligned}
\left\langle J^{i}\right\rangle & =-T_{0} \sum_{n} \operatorname{tr}\left[\sigma_{i} \mathcal{G}_{1}\left(\boldsymbol{x}, \boldsymbol{x}, \omega_{n}\right)\right], \\
\left\langle T_{0}{ }^{i}\right\rangle & =\left.T_{0} \sum_{n}\left[\frac{i}{4} e^{\sigma} \operatorname{tr}\left(\frac{\partial}{\partial x^{i}} \mathcal{G}_{1}\left(\boldsymbol{x}, \boldsymbol{x}^{\prime}, \omega_{n}\right)-\frac{\partial}{\partial x^{\prime i}} \mathcal{G}_{1}\left(\boldsymbol{x}, \boldsymbol{x}^{\prime}, \omega_{n}\right)\right)\right]\right|_{\boldsymbol{x}^{\prime}=\boldsymbol{x}}
\end{aligned}
$$




$$
\begin{aligned}
& +T_{0} \sum_{n}\left[\frac{1}{2} e^{\sigma} A_{i} \operatorname{tr} \mathcal{G}_{1}\left(\boldsymbol{x}, \boldsymbol{x}, \omega_{n}\right)+\frac{1}{4} e^{\sigma} \epsilon^{i j k} \partial_{j} \sigma \operatorname{tr}\left[\sigma_{k} \mathcal{G}_{0}\left(\boldsymbol{x}, \boldsymbol{x}, \omega_{n}\right)\right]\right] \\
& +T_{0} \sum_{n}\left[\frac{1}{2}\left(i \omega_{n}+A_{0}\right) \operatorname{tr}\left[\sigma_{i} \mathcal{G}_{1}\left(\boldsymbol{x}, \boldsymbol{x}, \omega_{n}\right)\right]-\frac{1}{2} e^{\sigma} a_{i}\left(i \omega_{n}+A_{0}\right) \operatorname{tr} \mathcal{G}_{1}\left(\boldsymbol{x}, \boldsymbol{x}, \omega_{n}\right)\right] \\
& +T_{0} \sum_{n}\left[-\frac{1}{8} e^{2 \sigma} \epsilon^{i j k} \partial_{j} a_{k} \operatorname{tr} \mathcal{G}_{0}\left(\boldsymbol{x}, \boldsymbol{x}, \omega_{n}\right)\right] .
\end{aligned}
$$

The traces that will be relevant for this computation are

$$
\begin{aligned}
\operatorname{tr} \mathcal{G}_{0}\left(\boldsymbol{x}, \boldsymbol{x}, \omega_{n}\right)= & -\frac{e^{-2 \sigma}}{4 \pi^{3 / 2}} \int_{0}^{\infty} \frac{d \rho}{\rho^{3 / 2}} e^{b^{2} \rho} \tilde{\omega}_{n} \\
\operatorname{tr}\left[\sigma_{i} \mathcal{G}_{1}\left(\boldsymbol{x}, \boldsymbol{x}, \omega_{n}\right)\right]= & \frac{1}{32 \pi^{3 / 2}} \int_{0}^{\infty} \frac{d \rho}{\rho^{3 / 2}} e^{b^{2} \rho} \epsilon^{i j k}\left[\partial_{j} a_{k}-8 \rho e^{-2 \sigma}\left(\partial_{j} A_{k}+A_{0} \partial_{j} a_{k}\right) \tilde{\omega}_{n}\right. \\
& \left.+6 \rho e^{-2 \sigma} \partial_{j} a_{k} \tilde{\omega}_{n}^{2}\right] \\
\operatorname{tr}\left(\frac{\partial}{\partial x^{i}} \mathcal{G}_{1}\left(\boldsymbol{x}, \boldsymbol{x}^{\prime}, \omega_{n}\right)-\right. & \left.\frac{\partial}{\partial x^{\prime i}} \mathcal{G}_{1}\left(\boldsymbol{x}, \boldsymbol{x}^{\prime}, \omega_{n}\right)\right)\left.\right|_{\boldsymbol{x}^{\prime}=\boldsymbol{x}}=\frac{i e^{-\sigma}}{8 \pi^{3 / 2}} \int_{0}^{\infty} \frac{d \rho}{\rho^{3 / 2}} e^{b^{2} \rho} \\
& \times \epsilon^{i j k}\left[2\left(\partial_{j} A_{k}+A_{0} \partial_{j} a_{k}\right)-\partial_{j} a_{k} \tilde{\omega}_{n}\right]
\end{aligned}
$$

where we have defined $\tilde{\omega}_{n} \equiv A_{0}+i \omega_{n}$. The remaining traces $\operatorname{tr} \mathcal{G}_{1}\left(\boldsymbol{x}, \boldsymbol{x}, \omega_{n}\right)$ and $\operatorname{tr}\left[\sigma_{i} \mathcal{G}_{0}\left(\boldsymbol{x}, \boldsymbol{x}, \omega_{n}\right)\right]$ vanish. After performing the summation over Matsubara frequencies as explained in appendix B and integrating in the proper time, this leads to the result

$$
\begin{aligned}
\left\langle J_{0}\right\rangle_{1} & =0, \\
\left\langle J^{i}\right\rangle_{1} & =e^{-\sigma} \epsilon^{i j k}\left[C A_{0} \partial_{j} A_{k}+\left(\frac{1}{2} C A_{0}^{2}+C_{2} T_{0}^{2}\right) \partial_{j} a_{k}\right], \\
\left\langle T_{00}\right\rangle_{1} & =0, \\
\left\langle T_{0}^{i}\right\rangle_{1} & =e^{-\sigma} \epsilon^{i j k}\left[\left(-\frac{C}{2} A_{0}^{2}+C_{2} T_{0}^{2}\right) \partial_{j} A_{k}+\left(-\frac{C}{6} A_{0}^{3}-C_{2} T_{0}^{2} A_{0}\right) \partial_{j} a_{k}\right],
\end{aligned}
$$

where $A_{k}=\mathcal{A}_{k}-A_{0} a_{k}$, and the constants take the values

$$
C=-\frac{1}{4 \pi^{2}}, \quad C_{2}=\frac{1}{24} .
$$

It has been indicated in [5] the possible appearance of contributions in eqs. (4.7) and (4.9) of the form $\left\langle J^{i}\right\rangle \sim C_{0} e^{-\sigma} \epsilon^{i j k} \partial_{j} A_{k}$ and $\left\langle T_{0}{ }^{i}\right\rangle \sim C_{1} e^{-\sigma} \epsilon^{i j k} \partial_{j} a_{k}$. These terms violate $\mathcal{C P} \mathcal{T}$ invariance, and our result leads correctly to a vanishing value for $C_{0}$ and $C_{1}$.

The method explained in previous sections makes use of Kaluza-Klein and gauge invariant quantities. As a consequence, the $\mathrm{U}(1)$ current that we obtain is the covariant current. It is related to the consistent current by [5]

$$
J^{\mu}=J_{\text {cons }}^{\mu}-\frac{C}{6} \epsilon^{\mu \nu \alpha \beta} \mathcal{A}_{\nu} \mathcal{F}_{\alpha \beta} .
$$

Note that the difference between consistent and covariant currents, which is the Bardeen polynomial, is only first order in derivatives [24]. Using eq. (4.11), the result for the 
consistent current at first order reads

$$
\begin{aligned}
\left\langle J_{\text {cons }, 0}\right\rangle_{1} & =-e^{\sigma} \epsilon^{i j k}\left[\frac{C}{3} A_{i} \partial_{j} A_{k}+\frac{C}{3} A_{0} A_{i} \partial_{j} a_{k}\right], \\
\left\langle J_{\text {cons }}^{i}\right\rangle_{1} & =e^{-\sigma} \epsilon^{i j k}\left[\frac{2}{3} C A_{0} \partial_{j} A_{k}+\left(\frac{1}{6} C A_{0}^{2}+C_{2} T_{0}^{2}\right) \partial_{j} a_{k}+\frac{C}{3} A_{k} \partial_{j} A_{0}\right] .
\end{aligned}
$$

Now, the general form of the consistent partition function at first order is [5]

$$
\mathcal{W}_{1}=\int d^{3} x \sqrt{g}\left[\alpha_{1}\left(\sigma, A_{0}\right) \epsilon^{i j k} A_{i} F_{j k}+\alpha_{2}\left(\sigma, A_{0}\right) \epsilon^{i j k} A_{i} f_{j k}+\alpha_{3}\left(\sigma, A_{0}\right) \epsilon^{i j k} a_{i} f_{j k}\right] .
$$

Using this formula in eqs. (2.11) and (2.12) and comparing with the results given by eqs. (4.8)-(4.9) and (4.12)-(4.13), one gets the following explicit expressions for the functions $\alpha_{i}\left(\sigma, A_{0}\right)$,

$$
\alpha_{1}\left(\sigma, A_{0}\right)=\frac{C}{6 T_{0}} A_{0}, \quad \alpha_{2}\left(\sigma, A_{0}\right)=\frac{1}{2}\left(\frac{C}{6 T_{0}} A_{0}^{2}+C_{2} T_{0}\right), \quad \alpha_{3}\left(\sigma, A_{0}\right)=0 .
$$

The coefficient $\alpha_{3}\left(\sigma, A_{0}\right)$ is proportional to $C_{1}$, which is zero in a $\mathcal{C P} \mathcal{T}$ invariant theory as mentioned above.

\section{Energy and charge density at second order. Renormalization}

In this section we will give the parts of $\left\langle J_{0}\right\rangle_{2}$ and $\left\langle T_{00}\right\rangle_{2}$ that are bilinear in derivatives of the background fields, i.e. contributions which are the product of first order terms, as well as the part proportional to the three-dimensional curvature. These expectation values follow from the equilibrium partition function which, in principle, can include all scalars containing two space derivatives. The explicit computation we will present shows that the four bilinear pseudo-scalars

$$
\epsilon^{i j k} \nabla_{i} \sigma f_{j k}, \quad \epsilon^{i j k} \nabla_{i} \sigma F_{j k}, \quad \epsilon^{i j k} \nabla_{i} A_{0} f_{j k}, \quad \epsilon^{i j k} \nabla_{i} A_{0} F_{j k}
$$

are absent. This is remarkable and requires an explanation. Under time reversal, the signature of $A_{0}$ and $\sigma$ is +1 , while that of $A_{i}$ and $a_{i}$ is -1 . As we have seen in the previous section, the consistent partition function exhibits a parity-odd dependence through the terms $\epsilon^{i j k} A_{i} F_{j k}$ and $\epsilon^{i j k} A_{i} f_{j k}$. According to this, the consistent partition function at first order does not change its sign under time reversal. On the other hand, the four pseudoscalars in (5.1) multiplied by any function of $A_{0}$ and $\sigma$ change their sign under $\mathcal{T}$, and it turns out that the parity violating partition function at second derivative order behaves in opposite way to that of first order. If follows that if the underlying Hamiltonian is invariant under $\mathcal{T}$, the parity violating part of the partition function at second order vanishes.

In the parity even sector the possible terms that can appear at second order have been classified in [22], and they are written in eq. (1.2). Our goal is to compute explicitly the coefficients $M_{i}, i=1, \ldots, 7$, for a free Weyl fermion and a massless Dirac fermion, in order to ascertain possible differences regarding the chiral anomaly at second order. 


\section{$5.1\left\langle T_{00}\right\rangle$ and $\left\langle J_{0}\right\rangle$ for Weyl fermions}

The evaluation of eqs. (2.7) and (2.9) for a chiral fermion with $\mathcal{G}_{2}\left(\boldsymbol{x}, \boldsymbol{x}, \omega_{n}\right)$ produces (see appendix B for details)

$$
\begin{aligned}
\left\langle J_{0}\right\rangle_{2}= & \frac{1}{24 \pi^{2}}\left(-\nabla^{i} A_{0} \nabla_{i} \sigma+\frac{1}{2} e^{2 \sigma} f_{i j} F^{i j}+\frac{1}{2} A_{0} e^{2 \sigma} f_{i j} f^{i j}\right) \mathcal{N}_{\Lambda}\left(\sigma, A_{0}\right) \\
& +\frac{1}{48 \pi^{2}}\left(\nabla^{i} A_{0} \nabla_{i} A_{0}+\frac{e^{2 \sigma}}{2} A_{0}^{2} f_{i j} f^{i j}+\frac{e^{2 \sigma}}{2} F_{i j} F^{i j}+e^{2 \sigma} A_{0} f_{i j} F^{i j}\right) \frac{\partial \mathcal{N}_{\Lambda}}{\partial A_{0}} \\
& -\frac{1}{24 \pi^{2}} A_{0} \nabla^{i} \sigma \nabla_{i} \sigma+\frac{7}{96 \pi^{2}} \nabla^{i} A_{0} \nabla_{i} \sigma+\frac{5}{192 \pi^{2}} e^{2 \sigma} f_{i j} F^{i j} \\
& +\frac{3}{64 \pi^{2}} e^{2 \sigma} A_{0} f_{i j} f^{i j}+\frac{A_{0}}{48 \pi^{2}} R, \\
\left\langle T_{00}\right\rangle_{2}= & \frac{1}{48 \pi^{2}}\left(\nabla^{i} A_{0} \nabla_{i} A_{0}+\frac{e^{2 \sigma}}{2} A_{0}^{2} f_{i j} f^{i j}+\frac{e^{2 \sigma}}{2} F_{i j} F^{i j}+e^{2 \sigma} A_{0} f_{i j} F^{i j}\right) \mathcal{N}_{\Lambda}\left(\sigma, A_{0}\right) \\
& +\left(\frac{A_{0}^{2}}{48 \pi^{2}}+\frac{T_{0}^{2}}{144}\right) \nabla^{i} \sigma \nabla_{i} \sigma-\frac{A_{0}}{12 \pi^{2}} \nabla^{i} A_{0} \nabla_{i} \sigma+\frac{5 e^{2 \sigma}}{64 \pi^{2}} A_{0} f_{i j} F^{i j} \\
& +\frac{7}{384 \pi^{2}}\left(2 \nabla^{i} A_{0} \nabla_{i} A_{0}+e^{2 \sigma} F_{i j} F^{i j}\right)+\left(\frac{19 A_{0}^{2}}{384 \pi^{2}}-\frac{T_{0}^{2}}{288}\right) e^{2 \sigma} f_{i j} f^{i j} \\
& -\left(\frac{A_{0}^{2}}{96 \pi^{2}}+\frac{T_{0}^{2}}{288}\right) R-\frac{23 e^{4 \sigma}}{3072 \pi^{2}} f_{i j} f^{i j} \int_{1 / \Lambda^{2}}^{\infty} \frac{d \rho}{\rho^{2}}+\frac{1}{4} e^{3 \sigma} \operatorname{rot} \boldsymbol{a} \cdot\langle\tilde{\boldsymbol{J}}\rangle_{1},
\end{aligned}
$$

where $\mathcal{N}_{\Lambda}\left(\sigma, A_{0}\right)$ turns out to be the following combination that includes vacuum and thermal effects in the massless case

$$
\mathcal{N}_{\Lambda}\left(\sigma, A_{0}\right) \equiv \int_{1 / \Lambda^{2}}^{1 / \Lambda_{I R}^{2}} \frac{d \rho}{\rho}+2 \sum_{n=1}^{\infty} \int_{0}^{1 / \Lambda_{I R}^{2}} \exp \left(-\frac{e^{2 \sigma} n^{2}}{4 T_{0}^{2} \rho}\right) \cos \left(n\left(\pi-A_{0} / T_{0}\right)\right)
$$

Note that the replacement $\Lambda \rightarrow \infty$ in the second integral is safe. We should note that both integrals are separately infrared divergent, but the summation in the thermal part removes the dependence on the IR regulator $\Lambda_{I R} \rightarrow 0$, so the leading logarithmic dependence of $\mathcal{N}_{\Lambda}$ is $\ln \left(e^{2 \sigma} \Lambda^{2} / T_{0}^{2}\right)$. A simple computation leads to

$$
\mathcal{N}_{\Lambda}\left(\sigma, A_{0}\right)=\ln \frac{e^{2 \sigma} \Lambda^{2}}{T_{0}^{2}}+\gamma_{E}-2 \ln 2+Q\left(\frac{A_{0}}{T_{0}}\right)
$$

where $Q(\nu)$ is the analytic continuation of the series

$$
Q(\nu)=-2 \sum_{n=1}^{\infty}(-1)^{n} \cosh (n \nu) \log \left(n^{2}\right) .
$$

Hence, $\partial \mathcal{N}_{\Lambda} / \partial A_{0}=T_{0}^{-1} Q^{\prime}(\nu)$, where $\nu=A_{0} / T_{0}$. Note that, although the last term of eq. (5.3) comes from the anomalous current of eq. (4.7), it produces an even parity contribution proportional to a combination of $f_{i j} f^{i j}$ and $f_{i j} F^{i j}$. Since other terms with this parametric dependence are already present in $T_{00}$, the contribution of the chiral anomaly at second order appears mixed with other parity even terms such as the coefficient of $\mathcal{N}_{\Lambda}$, which corresponds to the trace anomaly as we will see later. 


\subsection{Vacuum expectation values from regulators: Pauli-Villars regularization}

The vacuum contribution to the thermal expectation values is logarithmically divergent in the UV, so we need to choose a regularization procedure. This can be done in a gauge invariant way by means of Pauli-Villars regularization. In our case, it suffices to consider three heavy fermions with masses $M_{\ell}$ and weights $C_{\ell}$ obeying the conditions

$$
\begin{aligned}
& 1+\sum_{\ell=1}^{3} C_{\ell}=0, \\
& \sum_{\ell=1}^{3} C_{\ell} M_{\ell}^{2}=0 .
\end{aligned}
$$

A simple choice satisfying these constraints is $C_{1}=1, C_{2}=C_{3}=-1$, and $M_{1}=\sqrt{2} M$, $M_{2}=M_{3}=M$, where $M$ is a large mass.

For a massive fermion $\Psi_{\ell}$, the vacuum expectation values that result from eq. (2.1) by projecting on the left component read

$$
\begin{aligned}
\left\langle J_{0}\right\rangle_{2}^{\mathrm{vac}}= & \frac{1}{24 \pi^{2}}\left(-\nabla^{i} A_{0} \nabla_{i} \sigma+\frac{1}{2} e^{2 \sigma} f_{i j} F^{i j}+\frac{1}{2} A_{0} e^{2 \sigma} f_{i j} f^{i j}\right) \int_{1 / \Lambda^{2}}^{\infty} e^{-M_{\ell}^{2} \rho} \frac{d \rho}{\rho} \\
& -\frac{5}{96 \pi^{2}} \nabla^{i} A_{0} \nabla_{i} \sigma+\frac{1}{64 \pi^{2}}\left(e^{2 \sigma} f_{i j} F^{i j}+e^{2 \sigma} A_{0} f_{i j} f^{i j}\right) \\
\left\langle T_{00}\right\rangle_{2}^{\mathrm{vac}}= & {\left[\frac{1}{48 \pi^{2}}\left(\nabla^{i} A_{0} \nabla_{i} A_{0}+\frac{e^{2 \sigma}}{2} A_{0}^{2} f_{i j} f^{i j}+\frac{e^{2 \sigma}}{2} F_{i j} F^{i j}+e^{2 \sigma} A_{0} f_{i j} F^{i j}\right)\right.} \\
& \left.-\frac{11 M_{\ell}^{2}}{3072 \pi^{2}} e^{4 \sigma} f_{i j} f^{i j}\right] \int_{1 / \Lambda^{2}}^{\infty} e^{-M_{\ell}^{2} \rho} \frac{d \rho}{\rho}-\frac{23}{3072 \pi^{2}} e^{4 \sigma} f_{i j} f^{i j} \int_{1 / \Lambda^{2}}^{\infty} e^{-M_{\ell}^{2} \rho} \frac{d \rho}{\rho^{2}} \\
& +\frac{M_{\ell}^{2}}{64 \pi^{2}} e^{2 \sigma} \nabla^{i} \sigma \nabla_{i} \sigma+\frac{1}{64 \pi^{2}} \nabla^{i} A_{0} \nabla_{i} A_{0} \\
& -\frac{1}{384 \pi^{2}}\left(e^{2 \sigma} F_{i j} F^{i j}+2 e^{2 \sigma} A_{0} f_{i j} F^{i j}+e^{2 \sigma} A_{0}^{2} f_{i j} f^{i j}\right) \\
& +\frac{1}{192 \pi^{2}} e^{2 \sigma} R \int_{1 / \Lambda^{2}}^{\infty} M_{\ell}^{2} e^{-M_{\ell}^{2} \rho} \frac{d \rho}{\rho} .
\end{aligned}
$$

In the expression for $\left\langle T_{00}\right\rangle_{2}^{\text {vac }}$ we have not included the vacuum contribution from the term proportional to $\left\langle\Psi^{\dagger} \gamma^{i j} \Psi\right\rangle_{1}^{\text {vac }} f_{i j}$, which after projection on the left component, could combine with the last term of eq. (5.3) to give a possible finite part. However, such a finite part vanishes when the contributions from the physical field and the three regulators are combined:

$$
\int_{1 / \Lambda^{2}}^{\infty} \frac{d \rho}{\rho^{2}}+\sum_{\ell=1}^{3} C_{\ell} \int_{1 / \Lambda^{2}}^{\infty} e^{-M_{\ell}^{2} \rho} \frac{\left(1+M_{\ell}^{2} \rho\right)}{\rho^{2}} d \rho=-\left.\frac{e^{-2 M^{2} \rho}\left(-1+e^{M^{2} \rho}\right)^{2}}{\rho}\right|_{1 / \Lambda^{2} \rightarrow 0} ^{\infty}=0 .
$$

The finite terms independent on the mass are generated by the integrals

$$
\int_{0}^{\infty} e^{-M_{\ell}^{2} \rho} M_{\ell}^{2} d \rho=1, \quad \int_{0}^{\infty} e^{-M_{\ell}^{2} \rho} M_{\ell}^{4} \rho d \rho=1
$$


The use of the values of $C_{\ell}$ and $M_{\ell}$ given above yields the following combinations of integrals

$$
\begin{aligned}
\mathcal{N}_{\Lambda}+\sum_{\ell=1}^{3} C_{\ell} \int_{1 / \Lambda^{2}}^{\infty} e^{-M_{\ell}^{2} \rho} \frac{d \rho}{\rho} & =2 \gamma_{E}-3 \ln 2+\ln \frac{e^{2 \sigma} M^{2}}{T_{0}^{2}}+Q\left(\frac{A_{0}}{T_{0}}\right) \\
\sum_{\ell=1}^{3} C_{\ell} \int_{1 / \Lambda^{2}}^{\infty} M_{\ell}^{2} e^{-M_{\ell}^{2} \rho} \frac{d \rho}{\rho} & =-2 M^{2} \ln 2 \\
\int_{1 / \Lambda^{2}}^{\infty} \frac{d \rho}{\rho^{2}}+\sum_{\ell=1}^{3} C_{\ell} \int_{1 / \Lambda^{2}}^{\infty} e^{-M_{\ell}^{2} \rho} \frac{d \rho}{\rho^{2}} & =2 M^{2} \ln 2
\end{aligned}
$$

which, together with eq. (5.7), finally produce the total vacuum contribution of the PauliVillars regulators

$$
\begin{aligned}
\left\langle J_{0}\right\rangle_{2}^{\mathrm{PV}}= & \frac{1}{24 \pi^{2}}\left(-\nabla^{i} A_{0} \nabla_{i} \sigma+\frac{1}{2} e^{2 \sigma} f_{i j} F^{i j}+\frac{1}{2} A_{0} e^{2 \sigma} f_{i j} f^{i j}\right)\left(\ln \frac{e^{2 \sigma} \bar{M}^{2}}{T_{0}^{2}}+Q-\mathcal{N}_{\Lambda}\right) \\
& +\frac{5}{96 \pi^{2}} \nabla^{i} A_{0} \nabla_{i} \sigma-\frac{1}{64 \pi^{2}}\left(e^{2 \sigma} f_{i j} F^{i j}+e^{2 \sigma} A_{0} f_{i j} f^{i j}\right) \\
\left\langle T_{00}\right\rangle_{2}^{\mathrm{PV}}= & \frac{1}{48 \pi^{2}}\left(\nabla^{i} A_{0} \nabla_{i} A_{0}+\frac{e^{2 \sigma}}{2} A_{0}^{2} f_{i j} f^{i j}+\frac{e^{2 \sigma}}{2} F_{i j} F^{i j}+e^{2 \sigma} A_{0} f_{i j} F^{i j}\right) \\
& \times\left(\ln \frac{e^{2 \sigma} \bar{M}^{2}}{T_{0}^{2}}+Q-\mathcal{N}_{\Lambda}\right)+\frac{23}{3072 \pi^{2}} e^{4 \sigma} f_{i j} f^{i j} \int_{1 / \Lambda^{2}}^{\infty} \frac{d \rho}{\rho^{2}} \\
& -\frac{M^{2} \ln 2}{96 \pi^{2}} e^{2 \sigma} R-\frac{M^{2} \ln 2}{128 \pi^{2}} e^{4 \sigma} f_{i j} f^{i j}-\frac{1}{64 \pi^{2}} \nabla^{i} A_{0} \nabla_{i} A_{0} \\
& +\frac{1}{384 \pi^{2}}\left(e^{2 \sigma} F_{i j} F^{i j}+2 e^{2 \sigma} A_{0} f_{i j} F^{i j}+e^{2 \sigma} A_{0}^{2} f_{i j} f^{i j}\right)
\end{aligned}
$$

where we have defined the rescaled Pauli-Villars mass $\bar{M}=2^{-3 / 2} e^{\gamma_{E}} M$ to simplify the expressions. Thus, a renormalized expectation value is given by $\langle\mathcal{O}\rangle_{2}+\langle\mathcal{O}\rangle_{2}^{\mathrm{PV}}$, where the first summand is given either by eq. (5.2) or (5.3).

\section{Partition function at second order}

The general expression for the partition function at second order can be written as [22]

$$
\begin{aligned}
\mathcal{W}_{2}=\int d^{3} x \sqrt{g}[ & M_{1} g^{i j} \partial_{i} T \partial_{j} T+M_{2} g^{i j} \partial_{i} \nu \partial_{j} \nu+M_{3} g^{i j} \partial_{i} \nu \partial_{j} T \\
& \left.+T_{0}^{2} M_{4} f_{i j} f^{i j}+M_{5} F_{i j} F^{i j}+T_{0} M_{6} f_{i j} F^{i j}+M_{7} R\right],
\end{aligned}
$$

where $M_{i}=M_{i}(T, \nu)$, and

$$
T=T_{0} e^{-\sigma}, \quad \nu=\frac{A_{0}}{T_{0}}
$$


Using the variational formulae eqs. (2.11)-(2.12) with eq. (6.1), this gives

$$
\begin{aligned}
\left\langle J_{0}\right\rangle_{2}= & T_{0} e^{-\sigma}\left(e^{\sigma} M_{3}-T_{0} \frac{\partial M_{1}}{\partial \nu}+T_{0} \frac{\partial M_{3}}{\partial T}\right) \nabla^{i} \sigma \nabla_{i} \sigma \\
& -2 \frac{\partial M_{2}}{\partial T} \nabla^{i} \sigma \nabla_{i} A_{0}+\frac{e^{\sigma}}{T_{0}^{2}} \frac{\partial M_{2}}{\partial \nu} \nabla^{i} A_{0} \nabla_{i} A_{0}-\frac{\partial M_{5}}{\partial \nu} F_{i j} F^{i j} \\
& -T_{0} \frac{\partial M_{6}}{\partial \nu} f_{i j} F^{i j}-T_{0}^{2} \frac{\partial M_{4}}{\partial \nu} f_{i j} f^{i j}-e^{\sigma} \frac{\partial M_{7}}{\partial \nu} R, \\
\left\langle T_{00}\right\rangle_{2}= & -T_{0}^{3} e^{-2 \sigma}\left(2 e^{\sigma} M_{1}+T_{0} \frac{\partial M_{1}}{\partial T}\right) \nabla^{i} \sigma \nabla_{i} \sigma+2 T_{0}^{2} e^{-\sigma} \frac{\partial M_{1}}{\partial \nu} \nabla^{i} \sigma \nabla_{i} A_{0} \\
& +\left(\frac{\partial M_{2}}{\partial T}-\frac{\partial M_{3}}{\partial \nu}\right) \nabla^{i} A_{0} \nabla_{i} A_{0}+T_{0}^{2} \frac{\partial M_{5}}{\partial T} F_{i j} F^{i j}+T_{0}^{3} \frac{\partial M_{6}}{\partial T} f_{i j} F^{i j} \\
& +T_{0}^{4} \frac{\partial M_{4}}{\partial T} f_{i j} f^{i j}+T_{0}^{2} \frac{\partial M_{7}}{\partial T} R .
\end{aligned}
$$

By using the renormalized expressions of $\left\langle J_{0}\right\rangle_{2}$ and $\left\langle T_{00}\right\rangle_{2}$ computed in section 5 and plugging them into the l.h.s. of eqs. (6.3) and (6.4), one gets a system of 14 equations and 7 functions of two arguments. After solving these equations one gets the following result

$$
\begin{aligned}
M_{1}(T, \nu)= & -\frac{1}{144} \frac{1}{T}-\frac{1}{48 \pi^{2}} \frac{\nu^{2}}{T}, \\
M_{2}(T, \nu)= & \frac{1}{48 \pi^{2}} T\left(\ln \frac{\bar{M}^{2}}{T^{2}}+Q(\nu)-\frac{1}{4}-\frac{3}{4}\right), \\
M_{3}(T, \nu)= & -\frac{1}{12 \pi^{2}} \nu \\
M_{4}(T, \nu)= & -\frac{1}{96 \pi^{2}} \frac{\nu^{2}}{T}\left(\ln \frac{\bar{M}^{2}}{T^{2}}+Q(\nu)+\frac{11}{4}+6 \pi^{2} C+\frac{1}{4}\right)+\frac{1}{288} \frac{1}{T}-\frac{C_{2}}{8 T} \\
& +\frac{1}{384 \pi^{2}} \frac{1}{T^{3}} M^{2} \ln 2, \\
M_{5}(T, \nu)= & -\frac{1}{96 \pi^{2}} \frac{1}{T}\left(\ln \frac{\bar{M}^{2}}{T^{2}}+Q(\nu)-\frac{1}{4}+\frac{1}{4}\right), \\
M_{6}(T, \nu)= & -\frac{1}{48 \pi^{2}} \frac{\nu}{T}\left(\ln \frac{\bar{M}^{2}}{T^{2}}+Q(\nu)+\frac{7}{4}+6 \pi^{2} C+\frac{1}{4}\right) \\
M_{7}(T, \nu)= & -\frac{1}{288} T-\frac{1}{96 \pi^{2}} T \nu^{2}+\frac{1}{96 \pi^{2}} \frac{1}{T} M^{2} \ln 2 .
\end{aligned}
$$

The constants $C$ and $C_{2}$ are given by eq. (4.10), and $\bar{M}$ is defined after eq. (5.16). As we will see later the logarithmic dependence in $\bar{M}$ is related to conformal anomalies. The combination of terms proportional to $M^{2}$ in $M_{4}$ and $M_{7}$ is a pure renormalization effect. These terms can be renormalized by adding a counterterm proportional to the Ricci scalar $\tilde{R}$ of the $3+1$ dimensional metric,

$$
\mathcal{W}_{2}^{\mathrm{ct}}=-\frac{M^{2} \ln 2}{96 \pi^{2}} \int d^{4} x \sqrt{-G} \tilde{R}
$$

so that the renormalized partition function is $\mathcal{W}_{2}^{\text {ren }}=\mathcal{W}_{2}+\mathcal{W}_{2}^{\text {ct }}$. By using the relation between the scalar curvatures

$$
\tilde{R}=R+\frac{1}{4} e^{2 \sigma} f_{i j} f^{i j}-\frac{2 e^{-\sigma}}{\sqrt{g}} \partial_{i}\left(g^{i j} \sqrt{g} e^{\sigma} \partial_{j} \sigma\right),
$$


one gets

$$
\begin{aligned}
\mathcal{W}_{2}^{\mathrm{ct}}=-\frac{M^{2} \ln 2}{96 \pi^{2}}\left[\int d^{3} x \sqrt{g} \frac{e^{\sigma}}{T_{0}}\right. & \left(R+\frac{1}{4} e^{2 \sigma} f_{i j} f^{i j}\right) \\
& \left.-2 \int \frac{d^{3} x}{T_{0}} \sqrt{g} \frac{1}{\sqrt{g}} \partial_{i}\left(g^{i j} \sqrt{g} e^{\sigma} \partial_{j} \sigma\right)\right],
\end{aligned}
$$

where the last term vanishes. One can see that this counterterm exactly cancels the $M^{2}$ terms in $\mathcal{W}_{2}$. Then the renormalized coefficients $M_{4,7}^{\text {ren }}$ are the same as $M_{4,7}$, but removing the terms proportional to $M^{2}$.

The additive constants in $M_{2}, M_{4}, M_{5}$ and $M_{6}$, i.e. $-3 / 4,1 / 4,1 / 4$ and $1 / 4$, are the finite contributions coming from the Pauli-Villars regulator. We would like to emphasize that these contributions allow the thermal expectation values to be consistent with the partition function result. If they were not taken into account, them the system of equations for the functions $M_{i}(T, \nu)$ wouldn't have a solution for all the terms considered in $\left\langle J_{0}\right\rangle$ and $\left\langle T_{00}\right\rangle$. It is then remarkable that the vacuum contribution can affect the finite temperature part to make it consistent with the prediction from the partition function. Of course, for a given coefficient, e.g. $M_{5}$, we can remove some of these constants by a redefinition of $\bar{M}$, but the parametric dependence of the other coefficients on $M_{5}$ remains unaffected:

$$
\begin{aligned}
M_{2} & =-2 T^{2} M_{5}-\frac{T}{48 \pi^{2}} \\
M_{4}^{\text {ren }} & =\nu^{2} M_{5}+\frac{\left(1-36 C_{2}\right)}{288 T}-\frac{\left(1+2 \pi^{2} C\right) \nu^{2}}{32 \pi^{2} T}, \\
M_{6} & =2 \nu M_{5}-\frac{\left(1+3 \pi^{2} C\right) \nu}{24 \pi^{2} T} .
\end{aligned}
$$

Note that there are three independent combinations of $M_{2,4,5,6}$ which do not include logarithmic dependence in $\bar{M}$. They, or a linear combination of them, will appear in some of the transport coefficients of the hydrodynamic constitutive relations, see eq. (7.26).

To conclude this section, let us examine the transformation of the partition function given by eq. (6.1) under a Weyl rescaling. The different quantities involved transform under this as

$$
\begin{aligned}
g_{i j} & \rightarrow e^{2 \omega} g_{i j}, \quad g^{i j} \rightarrow e^{-2 \omega} g^{i j}, \quad \sigma \rightarrow \sigma+\omega, \quad \sqrt{g} \rightarrow e^{3 \omega} \sqrt{g}, \\
R & \rightarrow e^{-2 \omega}\left(R-2 g^{i j} \partial_{i} \omega \partial_{j} \omega-4 \nabla^{2} \omega\right),
\end{aligned}
$$

and the lower components $A_{i}, \mathcal{A}_{i}$, as well as $A_{0}$, are unchanged. Substituting into eq. (6.1), and using the formulae

$$
\left\langle T_{00}\right\rangle=-\frac{T_{0} e^{\sigma}}{\sqrt{g}} \frac{\delta \mathcal{W}_{2}}{\delta \sigma}, \quad\left\langle T^{i j}\right\rangle=-\frac{2 T}{\sqrt{g}} g^{i m} g^{j n} \frac{\delta \mathcal{W}_{2}}{\delta g^{m n}}
$$


we find the general form of the trace of the stress tensor at equilibrium,

$$
\begin{aligned}
\left.\frac{1}{\sqrt{g}} \frac{\delta \mathcal{W}_{2}}{\delta \omega}\right|_{\omega=0}= & \frac{1}{T}\left(g_{i j}\left\langle T^{i j}\right\rangle-e^{-2 \sigma}\left\langle T_{00}\right\rangle\right) \\
= & \left(M_{1}+T \frac{\partial M_{1}}{\partial T}-4 \frac{\partial^{2} M_{7}^{\text {ren }}}{\partial T^{2}}\right)(\nabla T)^{2} \\
& +\left(M_{3}+2 T \frac{\partial M_{1}}{\partial \nu}-8 \frac{\partial^{2} M_{7}^{\text {ren }}}{\partial T \partial \nu}\right) \nabla^{j} T \nabla_{j} \nu \\
& +\left(T \frac{\partial M_{3}}{\partial \nu}-4 \frac{\partial^{2} M_{7}^{\text {ren }}}{\partial \nu^{2}}\right)(\nabla \nu)^{2}+\left(M_{7}^{\text {ren }}-T \frac{\partial M_{7}^{\text {ren }}}{\partial T}\right) R \\
& +\left(T M_{3}-4 \frac{\partial M_{7}^{\text {ren }}}{\partial \nu}\right) \nabla^{2} \nu+\left(2 T M_{1}-4 \frac{\partial M_{7}^{\text {ren }}}{\partial T}\right) \nabla^{2} T \\
& +\left(M_{2}-T \frac{\partial M_{2}}{\partial T}\right)(\nabla \nu)^{2}-T_{0}^{2}\left(M_{4}^{\text {ren }}+T \frac{\partial M_{4}^{\text {ren }}}{\partial T}\right) f_{i j} f^{i j} \\
& -\left(M_{5}+T \frac{\partial M_{5}}{\partial T}\right) F_{i j} F^{i j}-T_{0}\left(M_{6}+T \frac{\partial M_{6}}{\partial T}\right) f_{i j} F^{i j} .
\end{aligned}
$$

The partition function is conformally invariant only if all the coefficients vanish. The first four lines in the last equality of eq. (6.20) only involve $M_{1}, M_{3}$ and $M_{7}^{\text {ren }}$, so the cancellation of the corresponding coefficients determines $M_{1}$ and $M_{3}$ in terms of $M_{7}^{\text {ren }}$,

$$
M_{1}=\frac{2}{T} \frac{\partial M_{7}^{\text {ren }}}{\partial T}, \quad M_{3}=\frac{4}{T} \frac{\partial M_{7}^{\text {ren }}}{\partial \nu},
$$

with $M_{7}^{\text {ren }}(T, \nu)=T f_{7}(\nu)$, where $f_{i}$ is an arbitrary function. The remainder conditions for conformal invariance leads to

$$
M_{2}=T f_{2}(\nu), \quad M_{4}^{\text {ren }}=T^{-1} f_{4}(\nu), \quad M_{5}=T^{-1} f_{5}(\nu), \quad M_{6}=T^{-1} f_{6}(\nu) .
$$

The model considered here only violates conformal invariance because renormalization effects, which lead to a logarithmic dependence on $\ln \frac{\bar{M}}{T}$ of $M_{2}, M_{4}^{\text {ren }}, M_{5}$ and $M_{6}$. In the case of a free Weyl fermion the anomalous partition function reads

$$
\begin{aligned}
\mathcal{W}_{\text {anom }}=\frac{1}{24 \pi^{2}} & \int d^{3} x \sqrt{g} \frac{1}{T} \ln \frac{\bar{M}}{T} \\
& \times\left(e^{-2 \sigma} g^{i j} \partial_{i} A_{0} \partial_{j} A_{0}-\frac{1}{2} A_{0}^{2} f_{i j} f^{i j}-\frac{1}{2} F_{i j} F^{i j}-A_{0} f_{i j} F^{i j}\right),
\end{aligned}
$$

which, by using the relation $A_{i}=\mathcal{A}_{i}-A_{0} a_{i}$, can be written only in terms of the four dimensional metric and the field strength of the gauge field $\mathcal{A}$ as

$$
\mathcal{W}_{\text {anom }}=\frac{1}{24 \pi^{2}} \int d^{3} x \sqrt{g} \frac{1}{T} \ln \frac{\bar{M}}{T} \times\left(-\frac{1}{2} G^{\mu \rho} G^{\nu \sigma} \mathcal{F}_{\mu \nu} \mathcal{F}_{\rho \sigma}\right)
$$

in agreement with the form of the local covariant action for the trace anomaly $[25,26]$

$$
\mathcal{W}_{\text {anom }}=c \int d^{4} x \sqrt{-G} \ln \frac{\bar{M}}{T} \mathcal{F}_{\mu \nu} \mathcal{F}^{\mu \nu}, \quad c=-\frac{1}{48 \pi^{2}} .
$$


The trace of the stress tensor for chiral fermions is given by

$$
G_{\mu \nu}\left\langle T^{\mu \nu}\right\rangle=-\frac{1}{48 \pi^{2}} \mathcal{F}_{\mu \nu} \mathcal{F}^{\mu \nu}
$$

The results presented above correspond to a free theory of one left Weyl fermion. The functions $M_{i}(T, \nu)$ obtained with one free Dirac fermion are twice the expressions (6.5)(6.11).

\section{Non-dissipative constitutive relations from the partition function}

In this section, we use the partition function of eq. (6.1) to determine partially the nondissipative part of the second order constitutive relations in terms of the functions $M_{i}(T, \nu)$. The stress tensor and charge current of the fluid may be written in the form

$$
\begin{aligned}
T^{\mu \nu} & =(\varepsilon+P) u^{\mu} u^{\nu}+P G^{\mu \nu}+T_{(1)}^{\mu \nu}+T_{(2)}^{\mu \nu}+\ldots, \\
J^{\mu} & =\rho u^{\mu}+J_{(1)}^{\mu}+J_{(2)}^{\mu}+\ldots,
\end{aligned}
$$

where $\varepsilon, P, \rho$ and $u^{\mu}$ are the energy density, pressure, charge density and local fluid velocity respectively. The subindex $(i)$ denotes the order in the derivative expansion. While the first order constitutive relations have been extensively considered in connection with the partition function, less attention has been paid to the study of the second order terms, at least in the case of a charged fluid. Here, we will restrict to the parity even terms that solely contain second order derivatives, i.e., terms of $I_{2}$ type in the notation of refs. [22, 27]. In the linearized theory of hydrodynamic fluctuations about the equilibrium these terms, together their parity odd counterparts, are the most important.

In general, the determination of non-dissipative parts in the constitutive relations at a given order can be made by the comparison of the corresponding value of $T^{\mu \nu}$ or $J^{\mu}$ evaluated at equilibrium with that obtained from the partition function. The outline of the procedure may be sketched by

$$
\left\langle\mathcal{O}_{i}\right\rangle_{\text {eq }}=\delta\left(\mathcal{O}_{\text {perfect fluid }}+\mathcal{O}_{1}+\ldots+\mathcal{O}_{i-1}\right)+\mathcal{O}_{i}
$$

where $\mathcal{O}_{k}$ corresponds to $T_{(k)}^{\mu \nu}$ or $J_{(k)}^{\mu}$. The left hand side is a specific variational derivative of the partition function, and $\delta\left(\mathcal{O}_{\text {perfect fluid }}+\ldots\right)$ is a correction of order $i$ due to all changes proportional to derivatives of the background that must be evaluated in the constitutive relations of lower orders. In the Landau frame we adopt, one also imposes the conditions

$$
T_{(i)}^{\mu \nu} u_{\nu}=0, \quad J_{(i)}^{\nu} u_{\nu}=0, \quad i=1,2, \ldots
$$

At the end, this procedure determines the transport coefficients, or the susceptibilities, in $\mathcal{O}_{i}$ in terms of functions appearing in the partition function. At first order, one finds [5]

$$
\begin{aligned}
& T_{(1)}^{\mu \nu}=0, \\
& J_{(1)}^{\mu}=\xi_{l} l^{\mu}+\xi_{B} \mathcal{B}^{\mu},
\end{aligned}
$$


where $l^{\mu}=\epsilon^{\mu \nu \rho \sigma} u_{\nu} \partial_{\rho} u_{\sigma}, \mathcal{B}^{\mu}=\frac{1}{2} \epsilon^{\mu \nu \rho \sigma} u_{\nu} \mathcal{F}_{\rho \sigma}$. Here $\delta \mathcal{O}_{\text {perfect fluid }}$ receives a correction of the fluid velocity $\left[\delta u_{(1)}\right]^{i}$, which at equilibrium is evaluated to a non-zero pseudo-vector, while $\delta T_{(1)}=\delta \mu_{(1)}=0$. With the notation of [21], the corresponding equations at second derivative order are

$$
\begin{aligned}
\left.T_{00}\right|_{\mathrm{eq}} & =\left[u_{(0)}\right]_{0}^{2} \delta \varepsilon_{(2)}+2(\varepsilon+P)\left[u_{(0)}\right]_{0}\left[\delta u_{(2)}\right]_{0}, \\
\left.T_{0}^{i}\right|_{\mathrm{eq}} & =(\varepsilon+P)\left[u_{(0)}\right]_{0}\left[\delta u_{(2)}\right]^{i}, \\
\left.T^{i j}\right|_{\mathrm{eq}} & =\delta P_{(2)} g^{i j}+(\varepsilon+P)\left[\delta u_{(1)}\right]^{i}\left[\delta u_{(1)}\right]^{j}+T_{(2)}^{i j}, \\
\left.J_{0}\right|_{\mathrm{eq}} & =\left[u_{(0)}\right]_{0} \delta \rho_{(2)}+\text { correction from } J_{(1)}, \\
\left.J^{i}\right|_{\mathrm{eq}} & =\rho\left[\delta u_{(2)}\right]^{i}+J_{(2)}^{i}+\text { correction from } J_{(1)},
\end{aligned}
$$

where

$$
\delta P_{(2)}=\frac{\partial P}{\partial \varepsilon} \delta \varepsilon_{(2)}+\frac{\partial P}{\partial \rho} \delta \rho_{(2)} .
$$

We have used some consequences of the Landau frame condition evaluated at equilibrium, which leads to $J_{(2) 0}=T_{(2) 00}=T_{(2) 0}{ }^{i}=0$, since $u_{(0)}^{\mu}=e^{-\sigma}(1,0,0,0)$. Note also that, since $\left[\delta u_{(1)}\right]^{i}$ is a pseudo-vector, the second order corrections that arise by substitution of $\left[\delta u_{(1)}\right]^{i}$ in the dissipative part of $T_{(1)}^{\mu \nu}$ are parity odd. The implications of these contributions for the parity odd transport coefficients have been recently studied in detail in ref. [21]. With regard to the charged current, the parts termed as corrections from $J_{(1)}$ are parity even, but the explicit form, that turns out to be quadratic in the anomaly coefficients, is not required for determining the linear terms in second derivatives.

The most general non-dissipative form of the stress tensor and charge current in the Landau frame at second order can be expressed as

$$
\begin{aligned}
T_{(2) \mu \nu}= & \Delta P\left(G_{\mu \nu}+u_{\mu} u_{\nu}\right)+T\left(\kappa_{1} \tilde{R}_{\langle\mu \nu\rangle}+\kappa_{2} u^{\alpha} u^{\beta} \tilde{R}_{\langle\mu \alpha \nu\rangle \beta}+\kappa_{3} \nabla_{\langle\mu} \nabla_{\nu\rangle} \nu\right) \\
& + \text { combination of six traceless bilinear tensors } \\
J_{(2) \mu}= & v_{1} P_{\mu \alpha} u_{\nu} \tilde{R}^{\nu \alpha}+v_{2} P_{\mu \alpha} \nabla_{\nu} \mathcal{F}^{\nu \alpha}+\text { combination of four bilinear vectors }
\end{aligned}
$$

where $\nu(x) \equiv \mu(x) / T(x)$ reduces in equilibrium to $A_{0}(\boldsymbol{x}) / T_{0}$, being $\mu(x)$ the chemical potential. ${ }^{1}$ The curvature quantities appearing in these constitutive relations are the Ricci and Riemann tensors of the four-dimensional background. The notation $X_{\langle\mu \nu\rangle}$ expresses the traceless and symmetric combination transverse to $u^{\mu}$,

$$
X_{\langle\mu \nu\rangle} \equiv P_{\mu}^{\alpha} P_{\nu}^{\beta}\left[\frac{1}{2}\left(X_{\alpha \beta}+X_{\beta \alpha}\right)-\frac{1}{3} G_{\alpha \beta} P^{\gamma \theta} X_{\gamma \theta}\right], \quad P_{\mu \nu} \equiv G_{\mu \nu}+u_{\mu} u_{\nu} .
$$

Although they do not play a role in our linear analysis, we also list the non-dissipative bilinear tensor and vector quantities [22] appearing in eq. (7.11),

$$
\begin{gathered}
\omega_{\langle\mu \alpha} \omega^{\alpha}{ }_{\nu\rangle}, \quad \omega_{\langle\mu \alpha} \mathcal{F}_{\nu\rangle}^{\alpha}, \quad \mathcal{F}_{\langle\mu \alpha} \mathcal{F}^{\alpha}{ }_{\nu\rangle}, \quad \nabla_{\langle\mu} T \nabla_{\nu\rangle} T, \quad \nabla_{\langle\mu} T \nabla_{\nu\rangle} \nu, \quad \nabla_{\langle\mu} \nu \nabla_{\nu\rangle} \nu, \\
P_{\mu}^{\alpha} P^{\beta \nu} \mathcal{F}_{\alpha \beta} \nabla_{\nu} T, \quad P_{\mu}{ }^{\alpha} P^{\beta \nu} \mathcal{F}_{\alpha \beta} \nabla_{\nu} \nu, \quad \omega_{\mu}{ }^{\alpha} \nabla_{\alpha} T, \quad \omega_{\mu}{ }^{\alpha} \nabla_{\alpha} \nu,
\end{gathered}
$$

\footnotetext{
${ }^{1}$ The notation for the coefficients $\kappa_{1}$ and $\kappa_{2}$ is like that of ref. [5].
} 
where $\omega_{\mu \nu}$ is the vorticity tensor

$$
\omega_{\mu \nu} \equiv \frac{1}{2} P_{\mu}^{\alpha} P_{\nu}^{\beta}\left(\nabla_{\alpha} u_{\beta}-\nabla_{\beta} u_{\alpha}\right),
$$

and $\mathcal{F}_{\mu \nu}$ is the gauge field strength. The correction to the pressure that includes second order derivatives with signature +1 under time reversal is given by the combination

$$
P_{(2)}=\kappa_{4} \tilde{R}+\kappa_{5} D^{2} T+\kappa_{6} D^{2} \nu
$$

where $\tilde{R}$ and $D^{2}$ are the scalar curvature and the Laplacian with respect to the fourdimensional metric $G^{\mu \nu}$, respectively. ${ }^{2}$

The goal is to determine the coefficients $\kappa_{i}$ and $\lambda_{j}$ in eq. (7.11) by comparison with the partition function. By using the following variational derivatives

$$
\begin{aligned}
\left.T_{00}\right|_{\text {eq }}= & -\frac{T_{0}^{2}}{T \sqrt{g}} \frac{\delta \mathcal{W}_{2}}{\delta \sigma} \\
= & T_{0}^{2}\left(-2 M_{1} \nabla^{2} T-M_{3} \nabla^{2} \nu+\frac{\partial M_{7}^{\text {ren }}}{\partial T} R\right)+\text { bilinear terms in derivatives }, \\
\left.T^{i j}\right|_{\text {eq }}= & -\frac{2 T}{\sqrt{g}} g^{i m} g^{j n} \frac{\delta \mathcal{W}_{2}}{\delta g^{m n}} \\
= & -2 T M_{7}^{\text {ren }}\left(R^{i j}-\frac{g^{i j}}{2} R\right)+2 T \frac{\partial M_{7}^{\text {ren }}}{\partial T}\left(\nabla^{i} \nabla^{j} T-g^{i j} \nabla^{2} T\right) \\
& +2 T \frac{\partial M_{7}^{\text {ren }}}{\partial \nu}\left(\nabla^{i} \nabla^{j} \nu-g^{i j} \nabla^{2} \nu\right)+\text { bilinear terms in derivatives }, \\
\left.T_{0}^{i}\right|_{\text {eq }}= & \frac{T}{\sqrt{g}}\left(\frac{\delta \mathcal{W}_{2}}{\delta a_{i}}-A_{0} \frac{\delta \mathcal{W}_{2}}{\delta A_{i}}\right) \\
= & 2 T T_{0}\left(2 \nu M_{5}-M_{6}\right) \nabla_{j} F^{j i}+2 T T_{0}^{2}\left(-2 M_{4}^{\text {ren }}+\nu M_{6}\right) \nabla_{j} f^{j i}+\ldots, \\
\left.J_{0}\right|_{\text {eq }}= & -\frac{T_{0}^{2}}{T \sqrt{g}} \frac{\delta \mathcal{W}_{2}}{\delta A_{0}} \\
= & \frac{T_{0}}{T}\left(M_{3} \nabla^{2} T+2 M_{2} \nabla^{2} \nu-\frac{\partial M_{7}^{\text {ren }}}{\partial \nu} R\right)+\ldots, \\
\left.J^{i}\right|_{\text {eq }}= & \frac{T}{\sqrt{g}} \frac{\delta \mathcal{W}_{2}}{\delta A_{i}} \\
= & -4 T M_{5} \nabla_{j} F^{j i}-2 T T_{0} M_{6} \nabla_{j} f^{j i}+\ldots,
\end{aligned}
$$

together with the formulae

$$
\begin{aligned}
\tilde{R}_{\langle i j\rangle} & =R_{i j}-\frac{g_{i j}}{3} R+\frac{1}{T} \nabla_{i} \nabla_{j} T-\frac{g_{i j}}{3} \frac{\nabla^{2} T}{T}+\ldots, \\
e^{-2 \sigma} \tilde{R}_{\langle i 0 j\rangle 0} & =-\frac{1}{T} \nabla_{i} \nabla_{j} T+\frac{g_{i j}}{3} \frac{\nabla^{2} T}{T}+\ldots, \\
\tilde{R} & =R+2 \frac{\nabla^{2} T}{T}+\ldots,
\end{aligned}
$$

\footnotetext{
${ }^{2}$ The quantity $D^{2} F$ becomes $\nabla^{2} F+g^{i j} \partial_{i} \sigma \partial_{j} F$ for a time-independent scalar field, so $\left.D^{2} F\right|_{\text {eq }}=\nabla^{2} F+$ bilinear terms in derivatives.
} 
we arrive at

$$
\begin{aligned}
\kappa_{1}= & -2 M_{7}^{\text {ren }}, \\
\kappa_{2}= & -2 M_{7}^{\text {ren }}-2 T \frac{\partial M_{7}^{\text {ren }}}{\partial T}, \\
\kappa_{3}= & 2 \frac{\partial M_{7}^{\text {ren }}}{\partial \nu}, \\
P_{(2)=} & \left(\frac{T M_{7}^{\text {ren }}}{3}-T^{2} \frac{\partial M_{7}^{\text {ren }}}{\partial T} \frac{\partial P}{\partial \varepsilon}-\frac{\partial M_{7}^{\text {ren }}}{\partial \nu} \frac{\partial P}{\partial \rho}\right) \tilde{R} \\
& +\left(-\frac{4 T}{3} \frac{\partial M_{7}^{\text {ren }}}{\partial \nu}+T^{2} M_{3} \frac{\partial P}{\partial \varepsilon}+2 M_{2} \frac{\partial P}{\partial \rho}\right) D^{2} \nu \\
& +\left[-\frac{2}{3} M_{7}^{\text {ren }}-\frac{4 T}{3} \frac{\partial M_{7}^{\text {ren }}}{\partial T}+2\left(T^{2} M_{1}+T \frac{\partial M_{7}^{\text {ren }}}{\partial T}\right) \frac{\partial P}{\partial \varepsilon}+\left(\frac{2}{T} \frac{\partial M_{7}^{\text {ren }}}{\partial \nu}+M_{3}\right) \frac{\partial P}{\partial \rho}\right] D^{2} T+\ldots
\end{aligned}
$$

Finally, by using the correction

$$
\left[\delta u_{(2)}\right]^{i}=-\left.\frac{1}{e^{\sigma}(\varepsilon+P)} T_{0}^{i}\right|_{\mathrm{eq}}
$$

and the comparison of the vectors in the charged current of eq. (7.11) with $\left.J^{i}\right|_{\text {eq }}$, we obtain

$$
\begin{aligned}
& v_{1}=4 T^{2}\left(2 \nu M_{5}-M_{6}\right)-\frac{8 \rho}{\varepsilon+P} T^{3}\left(M_{4}^{\mathrm{ren}}+\nu^{2} M_{5}-\nu M_{6}\right), \\
& v_{2}=-4 T M_{5}+\frac{2 \rho}{\varepsilon+P} T^{2}\left(2 \nu M_{5}-M_{6}\right) .
\end{aligned}
$$

Note that the transport coefficients do not depend on $T_{0}$, although $T_{0}$ appears explicitly in the partition function (6.1).

Eqs. (7.24) and (7.26) are general, and may be applied to the massless theory we have considered above. In this case we have $\partial P / \partial \varepsilon=1 / 3$ and $\partial P / \partial \rho=0$, so that the results in eqs. (6.5)-(6.11) produce $P_{(2)}=0$ to linear order. The effect of the trace anomaly appears in $P_{(2)}$ from

$$
\left.T^{i j}\right|_{\mathrm{eq}} g_{i j}-\left.T_{00}\right|_{\mathrm{eq}} e^{-2 \sigma}=T_{(2)}^{i j} g_{i j}=3 P_{(2)}=-\frac{1}{48 \pi^{2}} \mathcal{F}_{\alpha \beta} \mathcal{F}^{\alpha \beta},
$$

since the normalization condition $u^{\mu} u_{\mu}=-1$ implies that in equilibrium $\left[\delta u_{(1)}\right]_{0}=0$, and

$$
2 u_{(0)}^{0}\left[\delta u_{(2)}\right]_{0}+g_{i j}\left[\delta u_{(1)}\right]^{i}\left[\delta u_{(1)}\right]^{j}=0
$$

For the sake of completeness we show the explicit result of these transport coefficients obtained with the free field theory of Weyl fermions

$$
\begin{aligned}
\kappa_{1} & =\frac{T}{144}+\frac{1}{48 \pi^{2}} \frac{\mu^{2}}{T}, \\
\kappa_{2} & =2 \kappa_{1}
\end{aligned}
$$




$$
\begin{aligned}
& \kappa_{3}=-\frac{\mu}{24 \pi^{2}} \\
& v_{1}=\frac{1}{2}\left(C+\frac{1}{3 \pi^{2}}\right) \mu+\frac{\rho}{\varepsilon+P}\left[-\frac{1}{2}\left(C+\frac{1}{6 \pi^{2}}\right) \mu^{2}+\left(C_{2}-\frac{1}{36}\right) T^{2}\right], \\
& v_{2}=\frac{1}{24 \pi^{2}}\left(\ln \frac{\bar{M}^{2}}{T^{2}}+Q\left(\frac{\mu}{T}\right)\right)+\frac{\rho}{\varepsilon+P} \frac{1}{4}\left(C+\frac{1}{3 \pi^{2}}\right) \mu,
\end{aligned}
$$

where we have used that $\nu=\mu / T$. The only coefficient affecting second order derivatives which shows sensitivity to the renormalization scale is $v_{2}$ through their dependence on $-4 T M_{5}$. It is remarkable the absence of logarithms in $v_{1}$ and $\kappa_{1,2,3}$, which in the former case is a consequence of the particular combination of the $M$ 's. We also note the presence of $C$ and $C_{2}$ in these second order results.

The form of other second order non-dissipative coefficients at weak coupling at zero chemical potential, such as $\lambda_{3}$ and $\lambda_{4}$,

$$
T_{(2) \mu \nu}=T\left(\lambda_{3} \omega_{\langle\mu \alpha} \omega^{\alpha}{ }_{\nu\rangle}+\lambda_{4} \mathfrak{a}_{\langle\mu} \mathfrak{a}_{\nu\rangle}\right), \quad \mathfrak{a}_{\mu}=u^{\alpha} \nabla_{\alpha} u_{\mu},
$$

may be inferred from the results derived in section 5 of ref. [5]. There, the parametrization of the partition function is made through three functions $\tilde{P}_{i}(T)$, whose relation to the $M_{j}$ is

$$
\begin{aligned}
& \tilde{P}_{1}(T)=-2 M_{7}(T, \nu=0), \\
& \tilde{P}_{2}(T)=-2 M_{4}(T, \nu=0), \\
& \tilde{P}_{3}(T)=-2 T^{2} M_{1}(T, \nu=0),
\end{aligned}
$$

so, by using their formulae (5.8) and (5.15), one finds

$$
\begin{aligned}
& \kappa_{1}=-2 M_{7}, \\
& \kappa_{2}=-2 M_{7}-2 T M_{7}^{\prime}(T), \\
& \lambda_{3}=16 T^{2} M_{4}-6 M_{7}-2 T M_{7}^{\prime}(T), \\
& \lambda_{4}=-2 T^{2} M_{1}+4 T M_{7}^{\prime}(T)+2 T^{2} M_{7}^{\prime \prime}(T) .
\end{aligned}
$$

With the values at hand for the $M_{j}$, it turns out that $\lambda_{3}=\lambda_{4}=0$.

Let us compare these transport coefficients with some existing results in the literature. On the one hand $\kappa_{1}, \kappa_{2}$ and $\lambda_{3}$ have been explicitly computed in ref. [28] in the case of a conformal fluid at zero chemical potential. Our values for $\kappa_{1}$ and $\kappa_{2}$ after setting $\mu=0$ agree with the results in this reference. The constraint $\kappa_{2}=2 \kappa_{1}$ is also found in ref. [5]. Regarding $\lambda_{3}$, as mentioned above we get a vanishing value, and this is in contrast with the result obtained in ref. [28], where they find $\lambda_{3}^{\text {Moore,Sohrabi }}=-T^{2} / 24$ for a Weyl fermion, and $-T^{2} / 12$ for a Dirac fermion. The difference is related to the contribution proportional to $\operatorname{rot} \boldsymbol{a} \cdot\langle\tilde{\boldsymbol{J}}\rangle_{1}$ in eq. (5.3), which seems to be not included in the diagramatic computation of these authors. In this reference, triangle diagrams with cubic vertices in the fermion sector are only computed, while that contribution is tied to a three-point function from a seagull diagram with a quartic vertex. All the dependence on $C$ and $C_{2}$ in the second order coefficients comes from this term, and so we would expect that after removing these coefficients from the formulas, our result for $\lambda_{3}$ now agrees with the result in [28]. In fact 
after doing that, they agree modulo a numerical factor, $\left.\lambda_{3}\right|_{\mu=0, C=C_{2}=0}=-2 \lambda_{3}^{\text {Moore,Sohrabi }}$. This factor can only come from the coefficient multiplying the term $\propto 1 / T$ in $M_{4}^{\text {ren }}$, but after a careful check we have not detected any mistake in the computation.

Regarding the two terms involving the gauge field in eq. (7.11), an explicit computation of $\kappa_{3}$ has been performed in refs. [18, 29,30], and $v_{2}$ in ref. [18], in the context of a holographic model in 5 dimensions with pure gauge and mixed gauge-gravitational Chern Simons terms. Taking care of the different notation used in these references, one can make the identification $T \kappa_{3}=\Lambda_{5}$ and $v_{2}=-\xi_{10}$, where $\Lambda_{5}$ and $\xi_{10}$ are given by eqs. (7.26) and (4.38) of ref. [18] respectively. These coefficients receive contributions not induced by chiral anomalies, and so we cannot expect that the free field theory result of the present work agree with a strong coupling computation. However, it is tempting to study the parametric dependence in $\mu$ and $T$ of these coefficients. Using the results above for $\kappa_{3}$ and $v_{2}$, and the explicit expressions of refs. [18, 29, 30] for the analogous coefficients at strong coupling, one gets in the regime $\mu \ll T$

$$
T \kappa_{3}=-\frac{\mu T}{24 \pi^{2}} \propto \Lambda_{5}, \quad v_{2} \sim c(T)+\frac{5}{112 \pi^{4}} \frac{\mu^{2}}{T^{2}} \propto-\xi_{10},
$$

where in the free fermion computation $c(T)$ has a logarithmic dependence on $T$, while $c(T)$ is a constant in the holographic result, as the model of ref. [18] doesn't include conformal symmetry breaking effects. So, apart from these considerations, we can confirm agreement in the parametric dependence between both approaches.

\section{Conclusion}

In this paper we have addressed the computation of the thermal partition function of an ideal gas of massless fermions on an arbitrary stationary background in $3+1$ dimensions. Using a derivative expansion of the background fields, we have computed the equilibrium values of the charged $\mathrm{U}(1)$ current and energy-momentum tensor. We confirm the results previously reported in the literature for the parity odd transport coefficients at first order, and find as new results the parity even contributions at second order. From this, we derived the equilibrium partition function at second derivative order, and showed that the renormalization effects of the conformal anomaly mix with the chiral anomaly in some terms of the partition function. However this mixture does not appear in the constitutive relations. We have made the computation by using Pauli-Villars regularization. It is remarkable that the finite contributions from the regulators are crucial in order to obtain a consistent result for the partition function.

The equilibrium partition function can only account for non-dissipative effects, i.e. it makes contact with transport coefficients multiplying quantities that survive in equilibrium. While first-order non-dissipative coefficients, like the chiral magnetic and vortical conductivities, are $\mathcal{T}$-even and $\mathcal{P}$-odd, the situation at second order is however slightly different. Without violation of $\mathcal{T}$ invariance, the parity violating part of the partition function at second order vanishes, so the non-dissipative coefficients related to it are $\mathcal{T}$-even. We examined the constitutive relations in the Landau frame, and we derived the parametric 
dependence with temperature and chemical potential of five transport coefficients: $\kappa_{1,2,3}$ and $v_{1,2} . \kappa_{1}$ and $\kappa_{2}$ are consistent with a constraint previously reported in the literature, and the parametric dependence in temperature and chemical potential of $\kappa_{3}$ and $v_{2}$ agree with explicit results of these coefficients at strong coupling. We have evaluated also two additional coefficients at zero chemical potential, $\lambda_{3,4}$, and the result is that $\lambda_{4}$ is vanishing as required by conformal invariance, and a cancellation produces a zero value for $\lambda_{3}$.

\section{Acknowledgments}

We would like to thank Juan L. Mañes for discussions, collaboration on related topics, and for carefully reading the manuscript. This work has been supported by Plan Nacional de Altas Energías (FPA2011-25948 and FPA2012-34456), the Basque Government (IT559-10), Spanish MICINN Consolider-Ingenio 2010 Program CPAN (CSD2007-00042) and Centro de Excelencia Severo Ochoa Programme grant SEV-2012-0234. E.M. would like to thank the Universidad del País Vasco UPV/EHU, Spain, for their hospitality and support during the completion of parts of this work. The research of E.M. is supported by the Juan de la Cierva Program of the Spanish MINECO.

\section{A Free theory of Dirac fermions}

We show in this appendix some technical details of the free theory of Dirac fermions that are used in sections 2 and 3 . The action of the theory is

$$
S=\int d^{4} x \sqrt{-G} \mathcal{L}, \quad \text { where } \quad \mathcal{L}=-i \bar{\Psi} \underline{\gamma}^{\mu} \nabla_{\mu} \Psi+i m \bar{\Psi} \Psi
$$

where $\bar{\Psi}=\Psi^{\dagger} \gamma^{0}$. The space-time dependent Dirac matrices satisfy $\left\{\underline{\gamma}^{\mu}(x), \underline{\gamma}^{\nu}(x)\right\}=$ $2 G^{\mu \nu}(x)$, and they are related to the Minkowski matrices by $\underline{\gamma}^{\mu}(x)=e_{a}^{\mu}(x) \bar{\gamma}^{a}$, where $e_{a}^{\mu}(x)$ is the vierbein, $\left\{\gamma^{a}, \gamma^{b}\right\}=2 \eta^{a b}$ and $\eta^{a b}=\operatorname{diag}(-1,1,1,1)$. We choose the Minkowski matrices in the Weyl representation

$$
\gamma^{0}=\left(\begin{array}{cc}
\mathbb{O}_{2 \times 2} & \mathbb{1}_{2 \times 2} \\
-\mathbb{1}_{2 \times 2} & \mathbb{O}_{2 \times 2}
\end{array}\right), \quad \gamma^{i}=\left(\begin{array}{cc}
\mathbb{O}_{2 \times 2} & \sigma_{i} \\
\sigma_{i} & \mathbb{O}_{2 \times 2}
\end{array}\right), \quad i=1,2,3,
$$

where $\sigma_{i}$ are the Pauli matrices. The Dirac fields can be decomposed into left and right handed components, so that

$$
\Psi=\left(\begin{array}{c}
\psi_{L} \\
\psi_{R}
\end{array}\right), \quad \text { where } \quad \psi_{L(R)}=\left(\begin{array}{c}
\psi_{1} \\
\psi_{2}
\end{array}\right)_{L(R)} .
$$

$\psi_{L(R)}$ are left (right) Weyl fermions of two components. The covariant derivative of the Dirac field is given by

$$
\nabla_{\mu} \Psi=\left(\partial_{\mu}+\frac{1}{4} \omega_{\mu}^{a b} \gamma_{a b}-i \mathcal{A}_{\mu}\right) \Psi, \quad \gamma_{a b}=\frac{1}{2}\left[\gamma_{a}, \gamma_{b}\right]
$$


where, in the absence of torsion, the spin connection is related to the vierbein $e_{a}^{\nu}$ by

$$
\omega_{\mu}^{a b}=-e^{b \nu}\left(\partial_{\mu} e_{\nu}^{a}-\Gamma_{\mu \nu}^{\sigma} e_{\sigma}^{a}\right),
$$

and $\Gamma_{\mu \nu}^{\sigma}$ are the Christoffel symbols. The U(1) current and the energy-momentum tensor are defined respectively as

$$
J_{\mu}=\frac{1}{\sqrt{-G}} \frac{\delta S}{\delta \mathcal{A}^{\mu}}, \quad T^{\mu \nu}=\frac{e_{a}^{\nu}}{\sqrt{-G}} \frac{\delta S}{\delta e_{a \mu}} .
$$

These formulas yield the following expressions

$$
\begin{aligned}
J_{L, R}^{0} & =-\bar{\Psi} \underline{\gamma}^{0} \mathcal{P}_{L, R} \Psi, \quad J_{L, R}^{i}=-\bar{\Psi} \underline{\gamma}^{i} \mathcal{P}_{L, R} \Psi \\
T_{\mu \nu} & =\frac{i}{4} \bar{\Psi}\left[\underline{\gamma}_{\mu} \vec{\nabla}_{\nu}-\overleftarrow{\nabla}_{\nu} \underline{\gamma}_{\mu}+(\mu \leftrightarrow \nu)\right] \Psi
\end{aligned}
$$

where

$$
\bar{\Psi} \overleftarrow{\nabla}_{\mu}=\bar{\psi}\left(\overleftarrow{\partial}_{\mu}-\frac{1}{4} \omega_{\mu}^{a b} \gamma_{a b}+i \mathcal{A}_{\mu}\right)
$$

and the projectors on left and right handed components are defined as $\mathcal{P}_{L, R}=\frac{1}{2}\left(1 \pm \gamma_{5}\right)$. Using this technology, the explicit expressions of the currents and energy-momentum tensor can be obtained, and they are presented in section 2 .

\section{B Technical details on the computation of the thermal expectation val- ues: Matsubara sums}

Similarly as we do at first order in section 4 , the formulas for $\left\langle J_{0}\right\rangle$ and $\left\langle T_{00}\right\rangle$ at second order become

$$
\begin{aligned}
\left\langle J_{0}\right\rangle_{2} & =T_{0} \sum_{n}\left[-e^{\sigma} \operatorname{tr} \mathcal{G}_{2}\left(\boldsymbol{x}, \boldsymbol{x}, \omega_{n}\right)\right], \\
\left\langle T_{00}\right\rangle_{2} & =T_{0} \sum_{n}\left[e^{\sigma}\left(A_{0}+i \omega_{n}\right) \operatorname{tr} \mathcal{G}_{2}\left(\boldsymbol{x}, \boldsymbol{x}, \omega_{n}\right)-\frac{1}{4} e^{3 \sigma} \epsilon^{i j k} \partial_{j} a_{k} \operatorname{tr}\left[\sigma_{i} \mathcal{G}_{1}\left(\boldsymbol{x}, \boldsymbol{x}, \omega_{n}\right)\right]\right],
\end{aligned}
$$

where $\omega_{n}=\frac{2 \pi}{\beta}\left(n+\frac{1}{2}\right)$ are the fermionic Matsubara frequencies. The traces that will be relevant for this computation are

$$
\begin{aligned}
\operatorname{tr} \mathcal{G}_{2}\left(\boldsymbol{x}, \boldsymbol{x}, \omega_{n}\right)=\frac{e^{-2 \sigma}}{96 \pi^{3 / 2}} & \int_{0}^{\infty} \frac{d \rho}{\sqrt{\rho}} e^{b^{2} \rho}\left\{-2 \nabla^{i} \sigma \nabla_{i} \sigma\left[3 \tilde{\omega}_{n}+2 \rho e^{-2 \sigma} \tilde{\omega}_{n}^{3}\right]\right. \\
& +\nabla^{i} \sigma \nabla_{i} A_{0}\left[15+14 \rho e^{-2 \sigma} \tilde{\omega}_{n}^{2}\right] \\
& -\nabla^{i} A_{0} \nabla_{i} A_{0} \rho e^{-2 \sigma}\left[11 \tilde{\omega}_{n}+2 \rho e^{-2 \sigma} \tilde{\omega}_{n}^{3}\right] \\
& -\frac{1}{2} F_{i j} F^{i j} \rho\left[11 \tilde{\omega}_{n}+2 \rho^{2} e^{-2 \sigma} \tilde{\omega}_{n}^{3}\right] \\
& +f_{i j} F^{i j}\left[-e^{2 \sigma}-11 \rho A_{0} \tilde{\omega}_{n}+9 \rho \tilde{\omega}_{n}^{2}-2 \rho^{2} e^{-2 \sigma} A_{0} \tilde{\omega}_{n}^{3}+2 \rho^{2} e^{-2 \sigma} \tilde{\omega}_{n}^{4}\right]
\end{aligned}
$$




$$
\begin{aligned}
& +f_{i j} f^{i j}\left[-e^{2 \sigma} A_{0}+\frac{1}{16}\left(13 e^{2 \sigma}-88 \rho A_{0}^{2}\right) \tilde{\omega}_{n}+9 \rho A_{0} \tilde{\omega}_{n}^{2}\right. \\
& \left.-\frac{\rho}{8}\left(31+8 \rho e^{-2 \sigma} A_{0}^{2}\right) \tilde{\omega}_{n}^{3}+2 \rho^{2} e^{-2 \sigma} A_{0} \tilde{\omega}_{n}^{4}-\rho^{2} e^{-2 \sigma} \tilde{\omega}_{n}^{5}\right] \\
& \left.+\frac{1}{6} R\left[4 \tilde{\omega}_{n}-43 \rho e^{-2 \sigma} \tilde{\omega}_{n}^{3}-18 \rho^{2} e^{-4 \sigma} \tilde{\omega}_{n}^{5}\right]\right\},
\end{aligned}
$$

where $\tilde{\omega}_{n} \equiv A_{0}+i \omega_{n}$, in addition to $\operatorname{tr}\left[\sigma_{i} \mathcal{G}_{1}\left(\boldsymbol{x}, \boldsymbol{x}, \omega_{n}\right)\right]$ given by eq. (4.4). In these formulas $R$ is the Ricci scalar from $g_{i j}$.

The summations over Matsubara frequencies are performed in the following way. We define the function

$$
F\left(\rho, A_{0}\right):=T_{0} \sum_{n} e^{b^{2} \rho}=\frac{e^{-m^{2} \rho+\sigma}}{2 \sqrt{\pi \rho}} \vartheta_{3}\left(\frac{1}{2}\left(\pi-i A_{0} \beta\right), e^{-\frac{e^{2 \sigma} \beta^{2}}{4 \rho}}\right),
$$

where $b^{2}=-m^{2}+e^{-2 \sigma} \tilde{\omega}_{n}^{2}$ and $\vartheta_{3}$ is a Jacobi $\Theta$ function, which admits the expansion

$$
\vartheta_{3}(u, q)=1+2 \sum_{n=1}^{\infty} q^{n^{2}} \cos (2 n u) .
$$

Then the several powers in Matsubara frequencies

$$
F_{m}\left(\rho, A_{0}\right):=T_{0} \sum_{n} e^{b^{2} \rho} \tilde{\omega}_{n}^{m},
$$

can be obtained straightforwardly from appropriate combinations of derivatives of $F$. In particular, for the computation of the thermal expectation values of $J_{0}$ and $T_{00}$ up to second order in derivatives we need powers of $\tilde{\omega}_{n}$ up to order $m=6$. We find

$$
\begin{aligned}
& F_{1}\left(\rho, A_{0}\right)=\frac{e^{2 \sigma}}{2 \rho} \frac{\partial F}{\partial A_{0}}, \\
& F_{2}\left(\rho, A_{0}\right)=e^{2 \sigma}\left(\frac{\partial F}{\partial \rho}+m^{2} F\right), \\
& F_{3}\left(\rho, A_{0}\right)=\frac{e^{4 \sigma}}{2 \rho^{2}}\left(\rho \frac{\partial^{2} F}{\partial \rho \partial A_{0}}-\left(1-m^{2} \rho\right) \frac{\partial F}{\partial A_{0}}\right), \\
& F_{4}\left(\rho, A_{0}\right)=e^{4 \sigma}\left(\frac{\partial^{2} F}{\partial \rho^{2}}+2 m^{2} \frac{\partial F}{\partial \rho}+m^{4} F\right), \\
& F_{5}\left(\rho, A_{0}\right)=\frac{e^{6 \sigma}}{2 \rho^{3}}\left(\rho^{2} \frac{\partial^{3} F}{\partial \rho^{2} \partial A_{0}}-2 \rho\left(1-m^{2} \rho\right) \frac{\partial^{2} F}{\partial \rho \partial A_{0}}+\left(2-2 m^{2} \rho+m^{4} \rho^{2}\right) \frac{\partial F}{\partial A_{0}}\right), \\
& F_{6}\left(\rho, A_{0}\right)=\frac{e^{6 \sigma}}{4 \rho^{2}}\left(4 \rho^{2} \frac{\partial^{3} F}{\partial \sigma^{3}}+3 e^{2 \sigma} m^{2} \frac{\partial^{3} F}{\partial \rho \partial A_{0}^{2}}-30 m^{2} \rho \frac{\partial F}{\partial \sigma}\right. \\
& \left.\quad-2 m^{2}\left(3+12 m^{2} \rho-2 m^{4} \rho^{2}\right) F\right) .
\end{aligned}
$$

The term with the summation $\sum_{n=1}^{\infty}$ in the r.h.s. of eq. (B.5) is responsible for the finite temperature and chemical potential contributions in the thermal expectation values, and they are never affected by UV divergences when integrating in the proper time $\rho$. The 
other term " 1 " leads to the vacuum contributions which are affected by these divergences. In view of eqs. (B.4) and (B.7)-(B.12) it is clear that the vacuum contributions can only appear in those terms with even powers of $\tilde{\omega}_{n}$, as these terms include contributions with no derivatives with respect to $A_{0}$. Finally both vacuum and finite temperature contributions are affected by IR divergences when integrating in $\rho$, as explained in section 5 .

Open Access. This article is distributed under the terms of the Creative Commons Attribution License (CC-BY 4.0), which permits any use, distribution and reproduction in any medium, provided the original author(s) and source are credited.

\section{References}

[1] P. Kovtun, Lectures on hydrodynamic fluctuations in relativistic theories, J. Phys. A 45 (2012) 473001 [arXiv:1205.5040] [INSPIRE].

[2] P.B. Arnold, C. Dogan and G.D. Moore, The bulk viscosity of high-temperature QCD, Phys. Rev. D 74 (2006) 085021 [hep-ph/0608012] [INSPIRE].

[3] K. Fukushima, D.E. Kharzeev and H.J. Warringa, The chiral magnetic effect, Phys. Rev. D 78 (2008) 074033 [arXiv:0808.3382] [INSPIRE].

[4] D.T. Son and P. Surowka, Hydrodynamics with triangle anomalies, Phys. Rev. Lett. 103 (2009) 191601 [arXiv:0906.5044] [INSPIRE].

[5] N. Banerjee et al., Constraints on fluid dynamics from equilibrium partition functions, JHEP 09 (2012) 046 [arXiv: 1203.3544] [INSPIRE].

[6] K. Jensen et al., Towards hydrodynamics without an entropy current, Phys. Rev. Lett. 109 (2012) 101601 [arXiv:1203.3556] [INSPIRE].

[7] S. Bhattacharyya, Entropy current and equilibrium partition function in fluid dynamics, JHEP 08 (2014) 165 [arXiv: 1312.0220] [INSPIRE].

[8] S. Jeon and L.G. Yaffe, From quantum field theory to hydrodynamics: Transport coefficients and effective kinetic theory, Phys. Rev. D 53 (1996) 5799 [hep-ph/9512263] [INSPIRE].

[9] P.B. Arnold, G.D. Moore and L.G. Yaffe, Transport coefficients in high temperature gauge theories. 1. Leading log results, JHEP 11 (2000) 001 [hep-ph/0010177] [INSPIRE].

[10] P.B. Arnold, G.D. Moore and L.G. Yaffe, Effective kinetic theory for high temperature gauge theories, JHEP 01 (2003) 030 [hep-ph/0209353] [INSPIRE].

[11] K. Landsteiner, E. Megias and F. Pena-Benitez, Anomalous transport from Kubo formulae, Lect. Notes Phys. 871 (2013) 433 [arXiv:1207.5808] [InSPIRE].

[12] S. Bhattacharyya, V.E. Hubeny, S. Minwalla and M. Rangamani, Nonlinear fluid dynamics from gravity, JHEP 02 (2008) 045 [arXiv: 0712.2456] [INSPIRE].

[13] K. Landsteiner, E. Megias and F. Pena-Benitez, Gravitational anomaly and transport, Phys. Rev. Lett. 107 (2011) 021601 [arXiv:1103.5006] [INSPIRE].

[14] K. Landsteiner, E. Megias, L. Melgar and F. Pena-Benitez, Holographic gravitational anomaly and chiral vortical effect, JHEP 09 (2011) 121 [arXiv:1107.0368] [INSPIRE].

[15] S. Chapman, Y. Neiman and Y. Oz, Fluid/gravity correspondence, local wald entropy current and gravitational anomaly, JHEP 07 (2012) 128 [arXiv:1202.2469] [INSPIRE]. 
[16] M. Valle, Hydrodynamics in $1+1$ dimensions with gravitational anomalies, JHEP 08 (2012) 113 [arXiv: 1206.1538] [INSPIRE].

[17] K. Jensen, R. Loganayagam and A. Yarom, Thermodynamics, gravitational anomalies and cones, JHEP 02 (2013) 088 [arXiv: 1207.5824] [INSPIRE].

[18] E. Megias and F. Pena-Benitez, Holographic gravitational anomaly in first and second order hydrodynamics, JHEP 05 (2013) 115 [arXiv: 1304.5529] [INSPIRE].

[19] R. Banerjee, P. Chakraborty, S. Dey, B.R. Majhi and A.K. Mitra, Two dimensional hydrodynamics with gauge and gravitational anomalies, Phys. Rev. D 89 (2014) 104013 [arXiv: 1307.1313] [INSPIRE].

[20] D.E. Kharzeev and H.-U. Yee, Anomalies and time reversal invariance in relativistic hydrodynamics: the second order and higher dimensional formulations, Phys. Rev. D 84 (2011) 045025 [arXiv: 1105.6360] [INSPIRE].

[21] S. Bhattacharyya, J.R. David and S. Thakur, Second order transport from anomalies, JHEP 01 (2014) 010 [arXiv:1305.0340] [INSPIRE].

[22] S. Bhattacharyya, Entropy current from partition function: one example, JHEP 07 (2014) 139 [arXiv: 1403.7639] [INSPIRE].

[23] J.L. Mañes and M. Valle, Parity odd equilibrium partition function in $2+1$ dimensions, JHEP 11 (2013) 178 [arXiv:1310.2113] [INSPIRE].

[24] W.A. Bardeen and B. Zumino, Consistent and covariant anomalies in gauge and gravitational theories, Nucl. Phys. B 244 (1984) 421 [InSPIRE].

[25] M. Giannotti and E. Mottola, The trace anomaly and massless scalar degrees of freedom in gravity, Phys. Rev. D 79 (2009) 045014 [arXiv:0812.0351] [INSPIRE].

[26] C. Eling, Y. Oz, S. Theisen and S. Yankielowicz, Conformal anomalies in hydrodynamics, JHEP 05 (2013) 037 [arXiv: 1301.3170] [InSPIRE].

[27] S. Bhattacharyya, Constraints on the second order transport coefficients of an uncharged fluid, JHEP 07 (2012) 104 [arXiv: 1201.4654] [INSPIRE].

[28] G.D. Moore and K.A. Sohrabi, Thermodynamical second-order hydrodynamic coefficients, JHEP 11 (2012) 148 [arXiv:1210.3340] [INSPIRE].

[29] J. Erdmenger, M. Haack, M. Kaminski and A. Yarom, Fluid dynamics of R-charged black holes, JHEP 01 (2009) 055 [arXiv:0809.2488] [INSPIRE].

[30] N. Banerjee et al., Hydrodynamics from charged black branes, JHEP 01 (2011) 094 [arXiv: 0809.2596] [INSPIRE]. 